\title{
Article \\ FEEdBACk: An ICT-Based Platform to Increase Energy Efficiency through Buildings' Consumer Engagement
}

\author{
Filipe Soares ${ }^{1, *(\mathbb{D}}$, André Madureira ${ }^{1}\left(\mathbb{D}\right.$, Andreu Pagès ${ }^{2}$, António Barbosa ${ }^{1}\left(\mathbb{D}\right.$, António Coelho ${ }^{1}(\mathbb{D}$, \\ Fernando Cassola ${ }^{1}{ }^{(0)}$, Fernando Ribeiro ${ }^{1}{ }^{(0}$, João Viana ${ }^{1}$, José Andrade ${ }^{1}$, Marina Dorokhova ${ }^{3}{ }^{(0)}$, \\ Nélson Morais ${ }^{1}{ }^{\mathbb{D}}$, Nicolas Wyrsch ${ }^{3} \mathbb{D}$ and Trine Sørensen ${ }^{4}$
}

1 Institute for Systems and Computing Engineering, Tecnology and Science (INESC TEC), Campus da Faculdade de Engenharia da Universidade do Porto, Rua Dr. Roberto Frias, 4200-465 Porto, Portugal; andre.g.madureira@inesctec.pt (A.M.); antonio.c.barbosa@inesctec.pt (A.B.); acoelho@fe.up.pt (A.C.); fernando.c.marques@inesctec.pt (F.C.); fernando.j.ribeiro@inesctec.pt (F.R.); joao.p.viana@inesctec.pt (J.V.); jose.r.andrade@inesctec.pt (J.A.); nelson.a.morais@inesctec.pt (N.M.)

2 DEXMA, C/ Nàpols 189, Bajos D, 08013 Barcelona, Spain; apages@dexma.com

3 École Polytechnique fédérale de Lausanne (EPFL), STI IMT PV-LAB, MC A2 304 (Microcity), Rue de la Maladière 71b, CH-2002 Neuchâtel, Switzerland; marina.dorokhova@epfl.ch (M.D.); nicolas.wyrsch@epfl.ch (N.W.)

4 In-JeT ApS, Jeppe Aakjærs Vej, 153460 Birkerød, Denmark; tfs@in-jet.dk

* Correspondence: filipe.j.soares@inesctec.pt

\section{check for} updates

Citation: Soares, F.; Madureira, A.; Pagès, A.; Barbosa, A.; Coelho, A.; Cassola, F.; Ribeiro, F.; Viana, J.; Andrade, J.; Dorokhova, M.; Morais, N.; Wyrsch, N.; Sørensen, T. FEEdBACk: An ICT-Based Platform to Increase Energy Efficiency through Buildings' Consumer Engagement. Energies 2021, 14, 1524. https:// doi.org/10.3390/en14061524

Academic Editor: Mihaela Popescu

Received: 25 January 2021

Accepted: 2 March 2021

Published: 10 March 2021

Publisher's Note: MDPI stays neutral with regard to jurisdictional claims in published maps and institutional affiliations.

Copyright: (c) 2021 by the authors. Licensee MDPI, Basel, Switzerland. This article is an open access article distributed under the terms and conditions of the Creative Commons Attribution (CC BY) license (https:/ / creativecommons.org/licenses/by/ $4.0 /)$.

\begin{abstract}
Energy efficiency in buildings can be enhanced by several actions: encouraging users to comprehend and then adopt more energy-efficient behaviors; aiding building managers in maximizing energy savings; and using automation to optimize energy consumption, generation, and storage of controllable and flexible devices without compromising comfort levels and indoor air-quality parameters. This paper proposes an integrated Information and communications technology (ICT) based platform addressing all these factors. The gamification platform is embedded in the ICT platform along with an interactive energy management system, which aids interested stakeholders in optimizing "when and at which rate" energy should be buffered and consumed, with several advantages, such as reducing peak load, maximizing local renewable energy consumption, and delivering more efficient use of the resources available in individual buildings or blocks of buildings. This system also interacts with an automation manager and a users' behavior predictor application. The work was developed in the Horizon 2020 FEEdBACk (Fostering Energy Efficiency and BehAvioral Change through ICT) project.
\end{abstract}

Keywords: energy efficiency; ICT platform; gamification

\section{Introduction}

Buildings represent about $40 \%$ of the total energy demand in the European Union (EU) [1]. Energy efficiency is the aim of one of the 20-20-20 Europe 2020 targets; therefore, a broad area of research has been devoted to energy efficiency in buildings.

Energy consumption in buildings is influenced by six factors [2]: (1) climate, (2) envelope characteristics, (3) building-service and energy-system characteristics, (4) building operation and maintenance, (5) occupant activities and behavior, and (6) indoor environmental quality provided.

While climate is a factor that cannot be acted upon, several building envelope technologies can be adopted to improve a building's energy efficiency in the phase of construction or renovation of a building: air sealing, building insulation, windows, and roofs [3]. Factors (1) and (2) lie outside the scope of the FEEdBACk (Fostering Energy Efficiency and BehAvioral Change through ICT) project, which addresses the remaining ones. The factor buildingservice and energy-system characteristics has evolved due to advances in ICT; emerging concepts such as grid-responsive buildings play significant roles in stabilizing power grids 
for the future [4]. In FEEdBACk, tools deemed necessary in providing these services were developed, such as decision support tools for facility management and optimization tools for the use of controllable loads, which also serve the purpose of factor (4), building operation and maintenance. Motivating energy-efficient behaviors (factor (5)) and maintaining comfort levels (factor (6)) while monitoring both are also at the core of this project.

The main motivation behind this work is, therefore, to bridge two fields of knowledge that have been traditionally separate: social sciences and engineering. Most of the advanced engineering algorithms presented herein have been proposed elsewhere in the literature and have been proven to work in several contexts. However, the innovation of this project and contribution for the state-of-the-art does not lie in these algorithms individually, but rather their integration and application together with social sciences techniques to tackle a well-known problem that has a huge impact in global warming-the inefficient use of energy.

Several issues influence user behaviors, such as daily routines, economic factors, and ethics. Studies that were conducted to better understand consumer decision-making, and its behaviors $[5,6]$ demonstrate that users might change their behaviors if they perceive the real impact of their actions.

A recent systematic literature review conducted by Paone and Bacher [7] on the impact of building-occupant behaviors regarding energy efficiency and on its influencing methods identified three major stratagies to promote behavioral change: eco-feedback, social interaction, and gamification [8,9]. Although there is no standard definition for gamification, ultimately, various authors converge to define gamification as the use of game elements in non-game contexts to improve user experience and user engagement [10]. Regarding energy applications, major gamification techniques use game elements and mechanics to engage and motivate building occupants to adopt energy-efficient behaviors. Therefore, gamification becomes a central piece of the FEEdBACk project.

Besides FEEdBACk, a number of recent projects have aimed to increase energy efficiency through behavioral change using ICT solutions. Among these projects, the eTEACHER project (http:/ / www.eteacher-project.eu/, accessed on 15 February 2021) also focused on the end-user, used gamification techniques and sensors, and provided tailored advice to building users. However, with regard to engineering, no advanced algorithms such as the ones proposed in FEEdBACk were envisioned (e.g., none of their algorithms use forecasting techniques). Another noteworthy project is inBETWEEN (https:/ / cordis.europa.eu/project/id/768776 accessed on 15 February 2021), which also lacks the advanced algorithms proposed in FEEdBACk and has inferior use of sensors. Finally, in the UtilitEE project (https: / /www.utilitee.eu/ accessed on 15 February 2021), the building automation component dominates the project, but neither gamification nor algorithms using forecasting techniques were integrated. The remainder of this paper is as follows: in Section 2, the objectives, concepts, and methodology of FEEdBACk are provided; in Section 3 the data management and monitoring of energy and comfort are addressed; in Section 4, the segmentation of building user profiles are detailed; in Section 5, the gamification platform is presented; in Section 6, new ICT and data analysis applications developed in FEEdBACk are detailed; and in Section 7, conclusions are drawn and directions for future work are proposed.

\section{FEEdBACk Project}

FEEdBACk stands for Fostering Energy Efficiency and BehAvioral Change through ICT. The project was funded by the European Commission under Call "H2020-EE-20162017". In this section, an overview of the project objectives, methodology, and demonstration is presented.

The consortium is composed of 8 partners based in 7 different countries-Portugal, the Netherlands, Switzerland, Spain, the United Kingdom, Denmark, and Germany. In Appendix A a description of the involved partners is presented. 


\subsection{FEEdBACk Objectives}

The core objective of FEEdBACk is to promote, stimulate, and deliver energy efficiency through behavioral change. To encourage more efficient energy utilization and more responsible consumer behavior, a gamification platform was developed to motivate behavioral change by fostering awareness and consumer engagement through a pervasive application that analyzes context, sends personalized messages, and manages gamified peer competition and feedback.

The specific objectives are as follows:

- exploit innovative and user-friendly ICT solutions to promote users' and interested stakeholders' engagement and to foster energy efficiency;

- explore intelligent control and automation to optimize the utilization of controllable resources;

- include insights from social and behavioral sciences to maximize permanent behavioral change;

- maintain/improve comfort levels and indoor air quality;

- make energy-usage data accessible to users and to designated third parties;

- $\quad$ set up demonstrators with different characteristics, and quantify impacts using rigorous baselines; and

- investigate the potential for replication to other boundary conditions

\subsection{Concept and Methodology}

The FEEdBACk project envisages the development of innovative applications to encourage users of buildings to comprehend and then adopt more energy-efficient behaviors and to aid building managers in maximizing energy savings, not only fostering users' behavioral change but also using automation to optimize energy consumption, generation, and storage of controllable and flexible devices without compromising comfort levels and indoor air-quality parameters.

In order to achieve the project objectives, several components were developed. Figure 1 overviews the project components, which are detailed in the remainder of the present paper:

- $\quad$ FEEdBACk suite: Section 3.1

- $\quad$ Energy metering: Section 3.2

- $\quad$ Multisensor: Section 3.3

- User profile segmentation: Section 4

- Gamification platform: Section 5

- $\quad$ Net load forecasting and baseline apps: Section 6.1

- Load disaggregation app: Section 6.2

- Occupancy forecast app: Section 6.3

- $\quad$ Behavior predictor app: Section 6.4

- $\quad$ Energy manager app: Section 6.6

- $\quad$ Automation manager app: Section 6.5

The gamification platform is embedded in a broader ICT-based platform for energy efficiency with an interactive energy management system, which aids interested stakeholders in optimizing "when and at which rate" energy should be buffered and consumed, with several advantages, such as reducing peak load, maximizing local renewable energy consumption, and delivering more efficient use of the resources available in individual buildings or blocks of buildings. This system also interacts with an automation manager and a users' behavior predictor application.

The approach to motivate behavior change is made by intrinsically motivating the user to change procedures and to take advantage of opportunities to improve energy efficiency without compromising comfort level and autonomy. In order for the user to feel in control, the application analyzes the users' context and past behavior to choose the right moment to introduce advice notices. 
This pervasive application is composed of a context-aware module that identifies the context of the user (space and time) to provide input to a behavior predictor application. The behavior predictor incorporates advanced machine learning algorithms run in a closed loop to evaluate users' reactions to the stimulus sent by the energy manager. This application also provides a simple and intuitive interface for the gamification platform.

To maximize overall system energy efficiency, the energy manager also interacts with an automation manager application for which the main goal is to manage in an optimal manner all the controllable devices available in the buildings' premises, such as heating, ventilation, and air conditioning (HVAC) systems and thermal loads, taking into account equipment technical restrictions, comfort level, and indoor air-quality requirements.

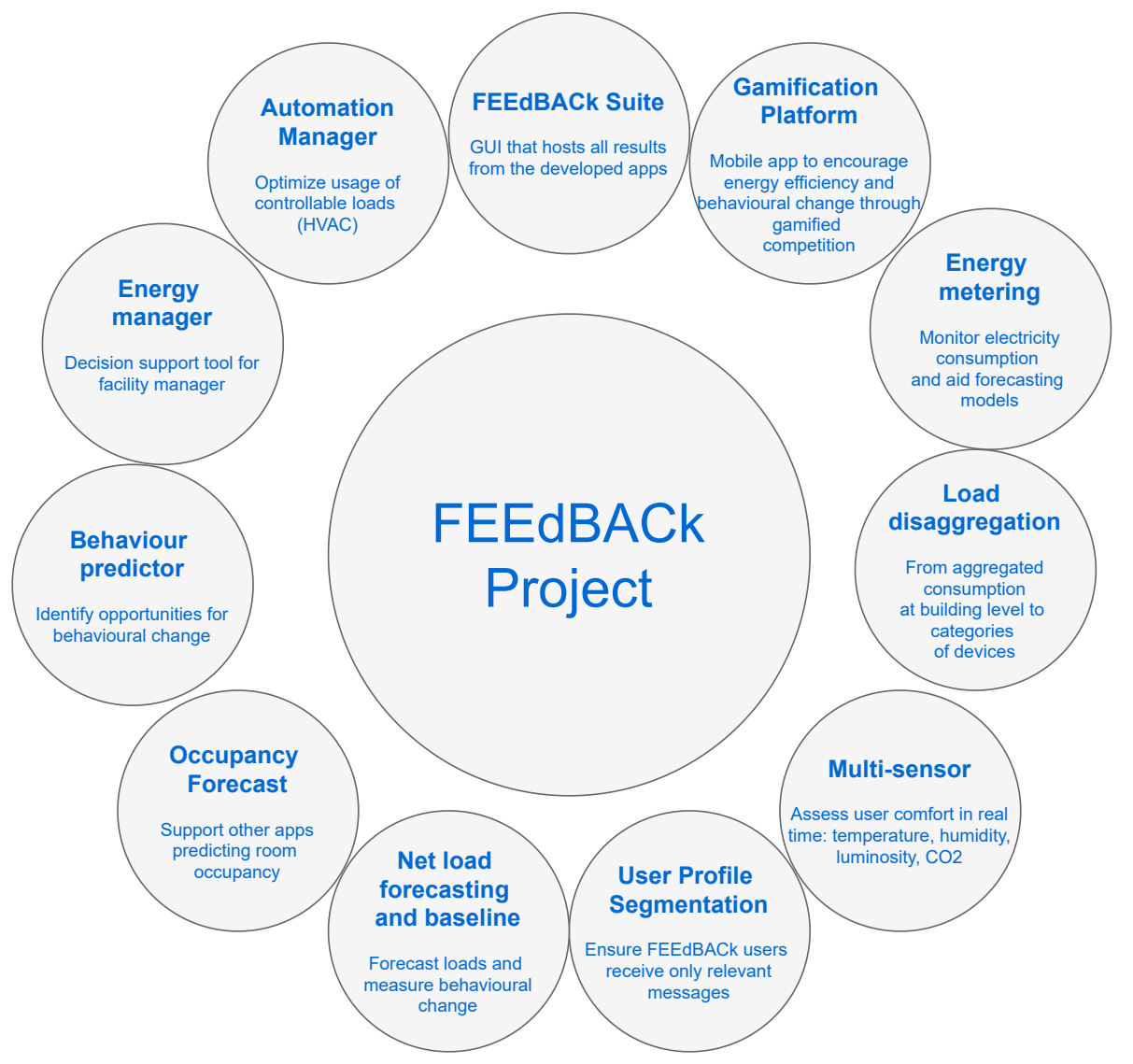

Figure 1. FEEdBACk suite integration block-scheme in a FEEdBACk ICT-based platform.

An overview of the demo sites (Portugal, Spain, and Germany) is provided in Appendix B.

The abovementioned apps were developed, tested, and implemented mostly in the Porto demo. Additionally, a competition envisioned by the project and partially implemented is described in Appendix C. However, due to the COVID-19 pandemic, the office and services buildings (Porto and Barcelona) were left empty for months whereas the residential building (Lippe) were occupied for longer hours than before the pandemic. Due to this reason, full implementation of the FEEdBACk solution was not completed, since the baseline scenario for energy consumption would not reflect the reality of the observed consumption, and therefore, the possible energy savings that would validate the FEEdBACk solution would not be realistic.

\section{Data Management Architecture for Monitoring Energy and Comfort}

\subsection{FEEdBACk Suite}

The FEEdBACk Suite is, as its name suggests, the FEEdBACk project apps' graphic user interface suite. It basically consists of a group of displays that aggregates the different 
results of the apps developed within the FEEdBACk project. The suite is placed over the DEXMA Monitoring platform as the company database is used by the project apps. Moreover, it is expected to let external third parties create their own apps and to use it as well.

The aim of the suite is to improve user experience and the tool's usability. Initially, the energy manager app had to display all these app results. However, that would not have been consistent with the energy manager app description and was not very intuitive for the user. For this reason, the consortium agreed on developing a display for each of the apps in the FEEdBACk project that were not displayed in the mobile app already.

The presented format allows the DEXMA platform to execute the installation process faster, simpler, and more efficiently while the end user is only required to click an install button (later, some extra configurations may be required for each app). The app's results displayed in the suite are as follows:

- $\quad$ Energy manager

- Net load forecasting

- Occupancy forecasting

- Load disaggregation

- Automation manager

In Figure 2, the whole FEEdBACk interconnection architecture is presented. It can be observed where the different apps are hosted and what is their relation with the ICTbased platform.

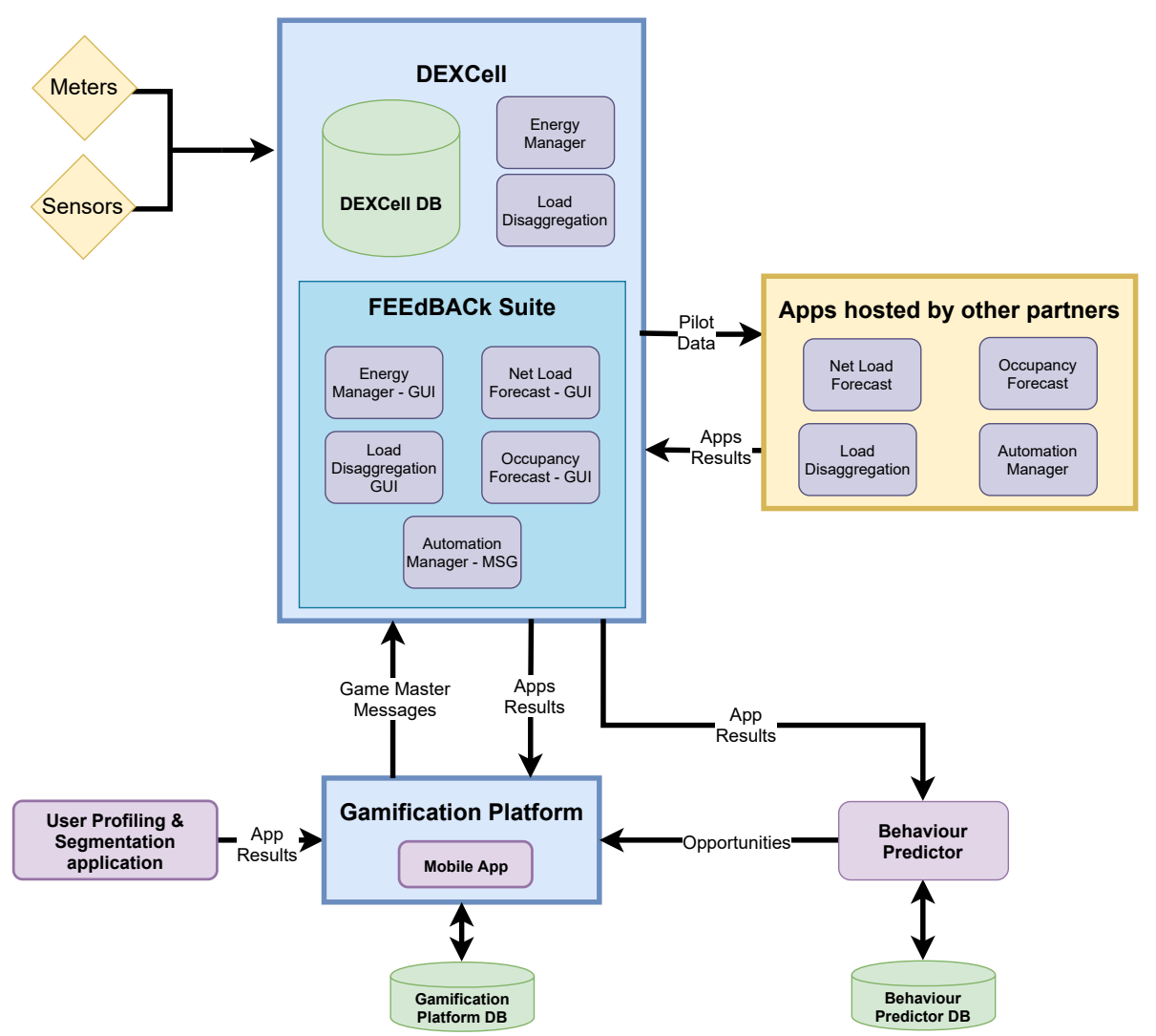

Figure 2. FEEdBACk Suite integration block-scheme in the FEEdBACk ICT-based platform.

As previously described in Section 3.1, an input for the Dexma Monitoring Platform is provided by energy meters and sensors. In the remainder of this section, an overview of the data management involving the meters and sensors used in the project is provided. 


\subsection{Metering}

This section concerns energy metering in the Porto demo (see Appendix B).

The building monitoring system consists of a group of meters addressing energy consumption in real-time in more than 160 points, installed in all circuit breakers on all electrical boards in the building. This building incorporates a very extensive set of loads and is equipped with an HVAC system that is centrally controlled, $4 \mathrm{~L}, 3$ server rooms (with about one hundred servers), around 400 personal computers, coffee machines, water machines, refrigerators, printers, and lights, among others.

The sub-metering system consists of three parts: energy meters, gateways, and a server. These equipment were used to measure the energy consumption in the most relevant circuits of the building. The energy meters were equipped with three current transformers (one per phase) and allowed us to obtain the following information:

- Active power

- Active energy

- Current

- Frequency

- Power factor

- Voltage

Every $15 \mathrm{~min}$, the meters store the measurements and then send it to a gateway, as shown in Figure 3.

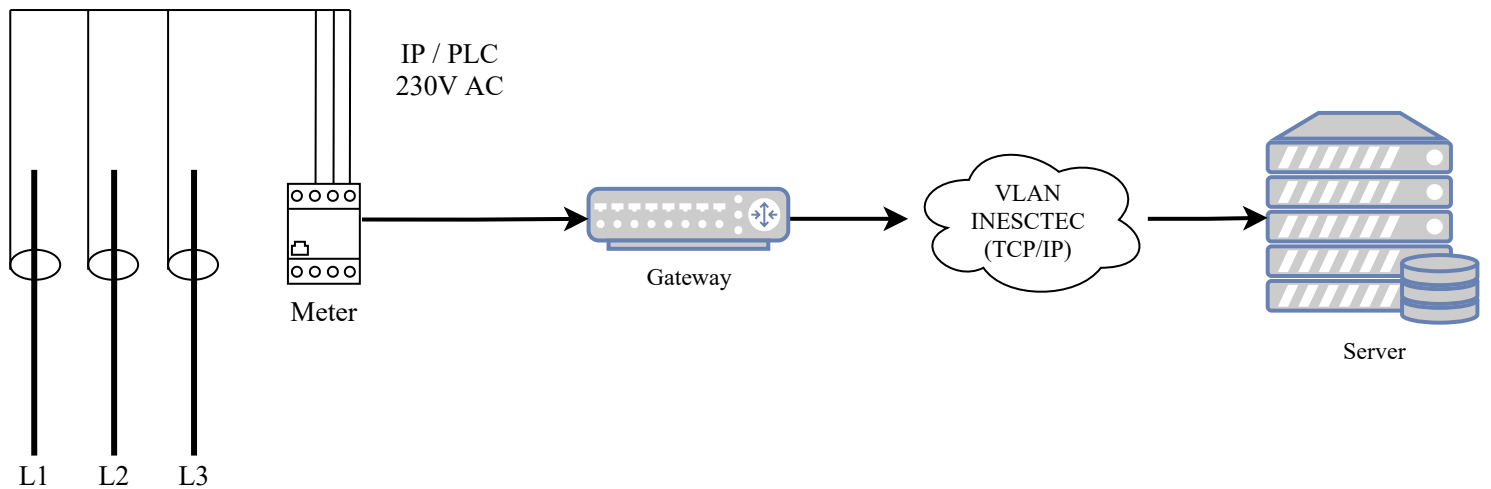

Figure 3. Measurement data flow.

This gateway's main function conveys the meter measurements to the server, where the data are permanently stored. The gateway is required since the meters use PLC (power-line communication) technology and the server only communicates using a TCP/IP (transmission control protocol/Internet protocol), commonly known as the computer network. This network uses the infrastructure previously implemented.

The server is a virtual machine running a Linux operating system, a MySQL database to allow us to persistently store all collected data, a JAVA plugin that performs the communication between the gateways, and the Perl scripting language to allow us to carry the data from the plugin into the database. The JAVA plugin collects information from all gateways and temporarily stores this information in object format that can be accessed through an HTTP REST API.

The current capacity of the server is about 250 thousand samplings, which, when divided by the approximately $160 \mathrm{~m}$ that make up the total installation, allows us to store temporarily about 1500 measurements per meter. If each meter provides a measurement every $15 \mathrm{~min}$, this allows us to store data for around 15 days.

Through a URL, it is possible to obtain the data of each meter in JSON format. To safeguard this information, a PERL script was developed that accesses the indicated URL, extracts this information, and inserts it in the database for permanent storage. 
To avoid duplicate values, whenever one accesses the REST API to obtain data, they are automatically deleted from the server. This script runs automatically at one-minute intervals. This enables the amount of data pending on the REST server to be as limited as possible and to make the data available as quickly as possible.

For the project competition in Porto, 14 teams were created (as detailed in Appendix C). They were distributed according to their location in the buildings and the ability to determine their consumption (e.g., one open space is one team, and a row of offices in the same area is another team). Generally, each circuit breaker is related to only one type of load, which can be divided into the following categories:

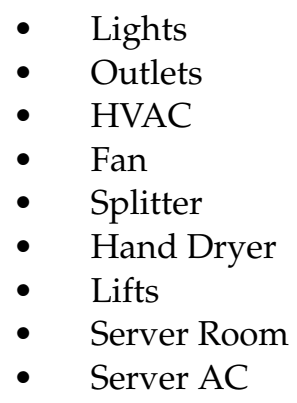

Due to limitations of existing electrical circuits and respective metering, it was necessary to divide the consumption into two teams in some cases. As it is not possible to physically perform a load division, it was necessary to create additional meters (which will be called virtual devices) that contain a percentage of the consumption obtained by the actual real meter. With the creation of virtual devices, the opportunity was taken to go further and to try to simplify the meter system to make it easier to share information between partners. Thus, virtual devices were renamed as follows EM_PLACE_LOAD, where the words are defined as follows:

- $\quad$ EM-electricity meter, to differentiate these devices from others;

- PLACE-load location in the building or related to the team; and

- LOAD—the type of load it represents.

Virtual devices were also created to represent the total consumption, either per floor, building, or team. In this way, it is possible to access information using just one meter, as if it was a real meter. These devices have the same periodic information as real meters and are generated shortly after obtaining information from real meters.

Table 1 lists the devices used by the gamification application, where it is possible to view part of the existing virtual devices:

Table 1. List of devices used by the gamification platform.

\begin{tabular}{ccccc}
\hline Team & Lights & Outlets & Fan & ALL \\
\hline inesc_tec_A_-1_Lab & EM_A-1_Lights & EM_A-1_Outlets & EM_A-1_Fan & EM_A-1_ALL \\
inesc_tec_A_1_OF & EM_A1_OF_Lights & EM_A1_OF_Outlets & EM_A1_OF_Fan & EM_A1_OF_ALL \\
inesc_tec_A_1_OS & EM_A1_OS_Lights & EM_A1_OS_Outlets & EM_A1_OS_Fan & EM_A1_OS_ALL \\
inesc_tec_A___OF & EM_A2_OF_Lights & EM_A2_OF_Outlets & EM_A2_OF_Fan & EM_A2_OF_ALL \\
inesc_tec_A_2_OS & EM_A2_OS_Lights & EM_A2_OS_Outlets & EM_A2_OS_Fan & EM_A2_OS_ALL \\
inesc_tec_A_3_OF & EM_A3_OF_Lights & EM_A3_OF_Outlets & EM_A3_OF_Fan & EM_A3_OF_ALL \\
inesc_tec_A_3_OS & EM_A3_OS_Lights & EM_A3_OS_Outlets & EM_A3_OS_Fan & EM_A3_OS_ALL \\
inesc_tec_A_4 & EM_A4_Lights & EM_A4_Outlets & EM_A4_Fan & EM_A4_ALL \\
inesc_tec_B_-1_Lab & EM_B-1_LAB_Lights & EM_B-1_LAB_Outlets & EM_B-1_LAB_Fan & EM_B-1_LAB_ALL \\
inesc_tec_B_1_OF & EM_B1_OS_Lights & EM_B1_OF_Outlets & EM_B1_OF_Fan & EM_B1_OF_ALL \\
inesc_tec_B_1_OS & EM_B2_OF_Lights & EM_B1_OS_Outlets & EM_B1_OS_Fan & EM_B1_OS_ALL \\
inesc_tec_B_2_OF & EM_B2_OS_Lights & EM_B2_OF_Outlets & EM_B2_OF_Fan & EM_B2_OF_ALL \\
inesc_tec_B_2_OS & EM_B2_OF_Lights & EM_B2_OS_Outlets & EM_B2_OS_Fan & EM_B2_OS_ALL \\
inesc_tec_B_4_OS & EM_B4_OS_Lights & EM_B4_OS_Outlets & EM_B4_OS_Fan & EM_B4_OS_ALL \\
\hline
\end{tabular}


These data were sent via HTTP REST API in JSON format to DEXCell (as detailed in Section 3.1), thus allowing us to share information with other partners not only through custom graphics but also through its own API. Figure 4 represents an example of the data collected from meters and visualized using the FEEdBACk suite presented in Section 3.1.

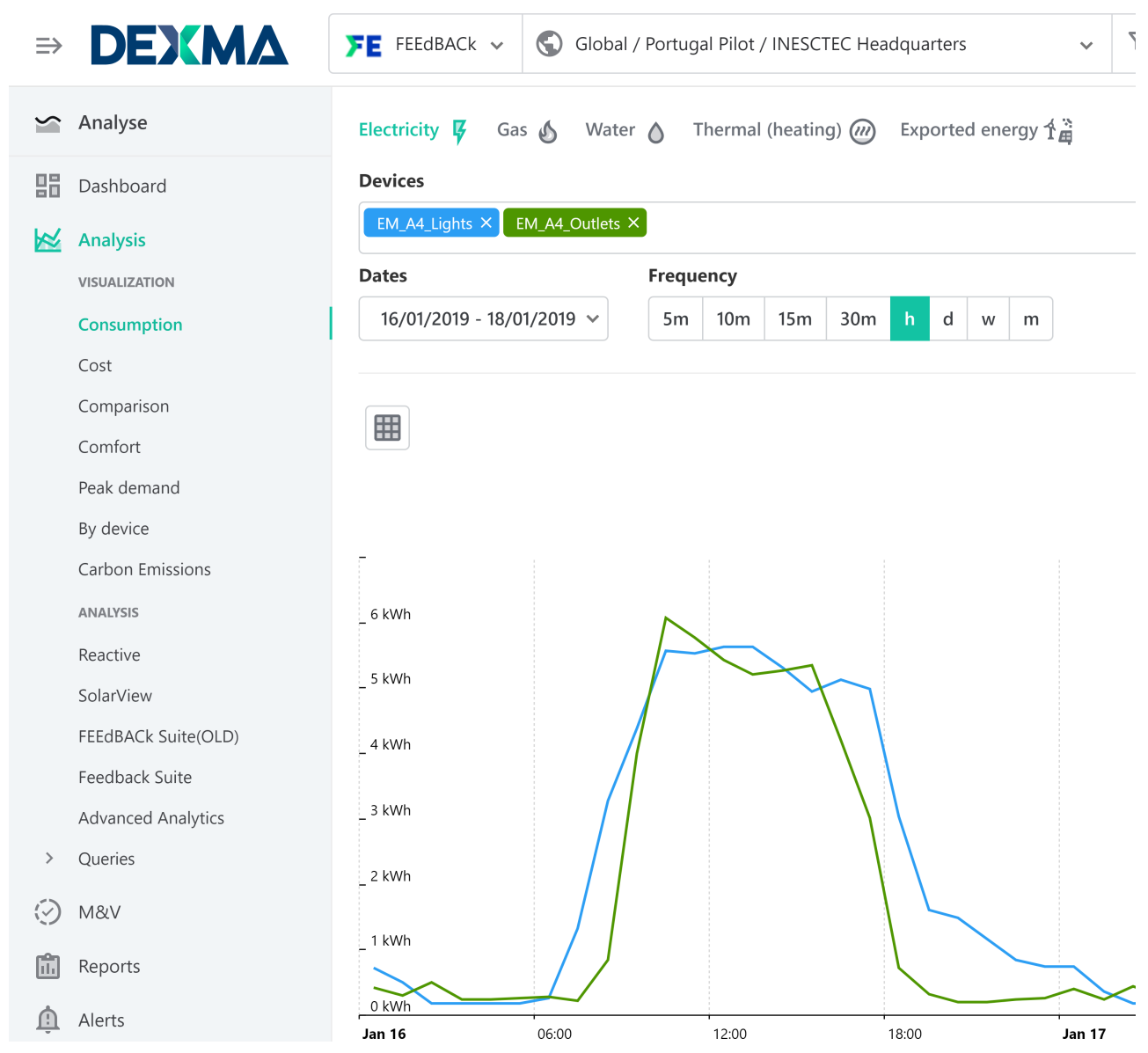

Figure 4. Chart with energy metering obtained from the DEXCell platform (Dexma partner); see Section 3.1.

\subsection{Multisensor}

In order to assess the indoor air quality in a workspace, a sensor was installed that is capable of measuring temperature, humidity, and $\mathrm{CO}_{2}$. Furthermore, it was necessary to be able to send measurements in real time using the existing infrastructure (typically wireless network) and to store this data persistently in a database.

Obtaining these data has three objectives:

1. Inform users in real-time about the current conditions of their room,

2. Allow us to define the comfort values for each room (considering that each room has different sizes, orientations, and number of users),

3. Allow us to create forecasting models regarding the occupation of the locations where they are installed-see the sections regarding occupancy forecast (Section 6.3) and behavior predictor (Section 6.4).

As there was not an ideal, off-the-shelf solution that could suit the requirements presented above, we decided to design a sensor from scratch with the following requirements:

- $\quad$ Sensors: Temperature, Humidity, and $\mathrm{CO}_{2}$

- Real time communication

- Wireless network connection

- Ability to store measurements locally in case of network failure 
- Ability to send those measurements when connectivity is restored

- Possibility to define how often to send measures

- Possibility to use rechargeable battery

- Direct power supply (typically $230 \mathrm{~V}$ )

- Configuration via web page (from the device itself)

The sensor is shown in Figure 5.

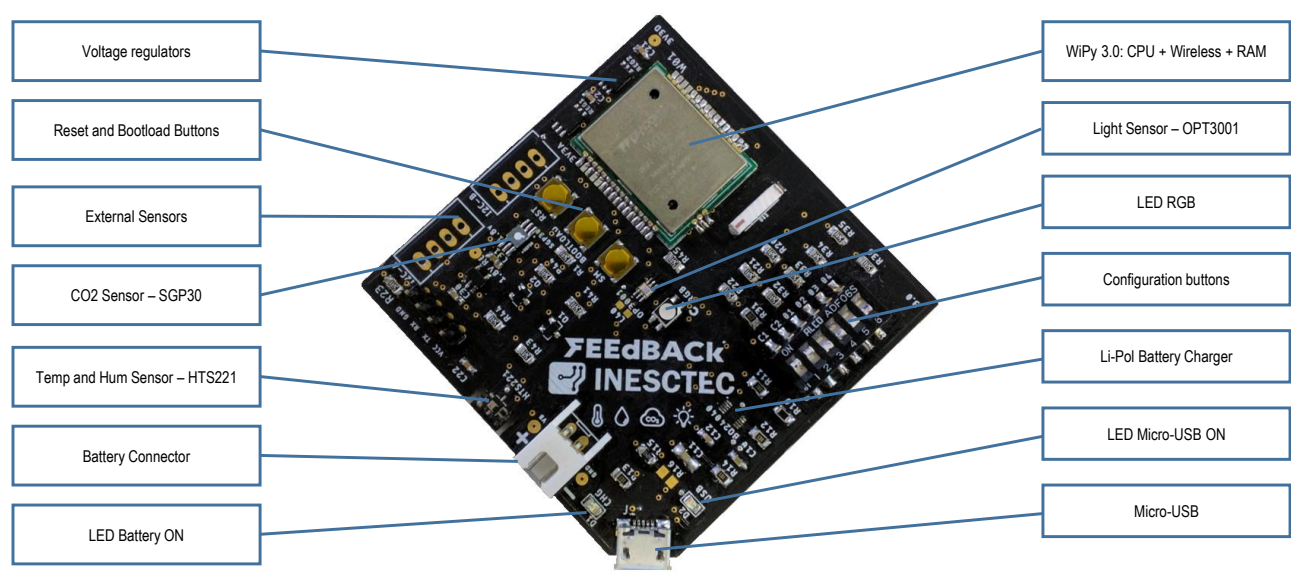

Figure 5. Multisensor developed for FEEdBACk.

Basically, the sensor consists of a WiPy processor, measurement sensors, micro-USB port , and battery connector for power. During the development stage of the sensor, we decided to include a light sensor since the extra cost was relatively small.

The sensor operates as follows:

- Every $15 \mathrm{~min}$, the micro-python processor checks all the sensors for their measurements,

- It sends this information via a web service to a server and stores the information in a MySQL database;

- This information is then sent to the DEXCell project platform.

Between the measurement period, the sensors enters a state called "deep sleep", where their energy consumption is reduced to a minimum and only keeps a timer running that allows the device to wake up again after a predefined period. This solution allows the device to be used with a $2000 \mathrm{mAh}$ lithium battery. With this system, it is possible to have the device unplugged and running during circa one month and a half without recharging needs. Due to physical impossibility, it is not possible to know the battery's state of charge, so it is not possible to know when to replace it with a charged one. Another limitation occurs when it operates continuously (or at relatively short intervals). The processor has a tendency to heat up, which will influence the measured temperature value. Another limitation exists on the $\mathrm{CO}_{2}$ sensor, since it does not read values below $400 \mathrm{ppm}$. In general terms, this not a concern since the discomfort limits are the reason for measuring $\mathrm{CO}_{2}$ and those are only above $600 \mathrm{ppm}$. However, the information received by users may seem inaccurate because it may give rise to the idea that the sensor has malfunctioned (always transmitting the same value) when the sensor does not actually detect lower values. Regarding the light sensor, it was found that it obtains different values depending on the location of the equipment. Moreover, it makes no distinction between sunlight or artificial light. However, it is an important aid in occupancy charts as the room is typically not used without light.

Figures 6 and 7 show the measurements obtained by the multisensor on 24 September 2020 . 


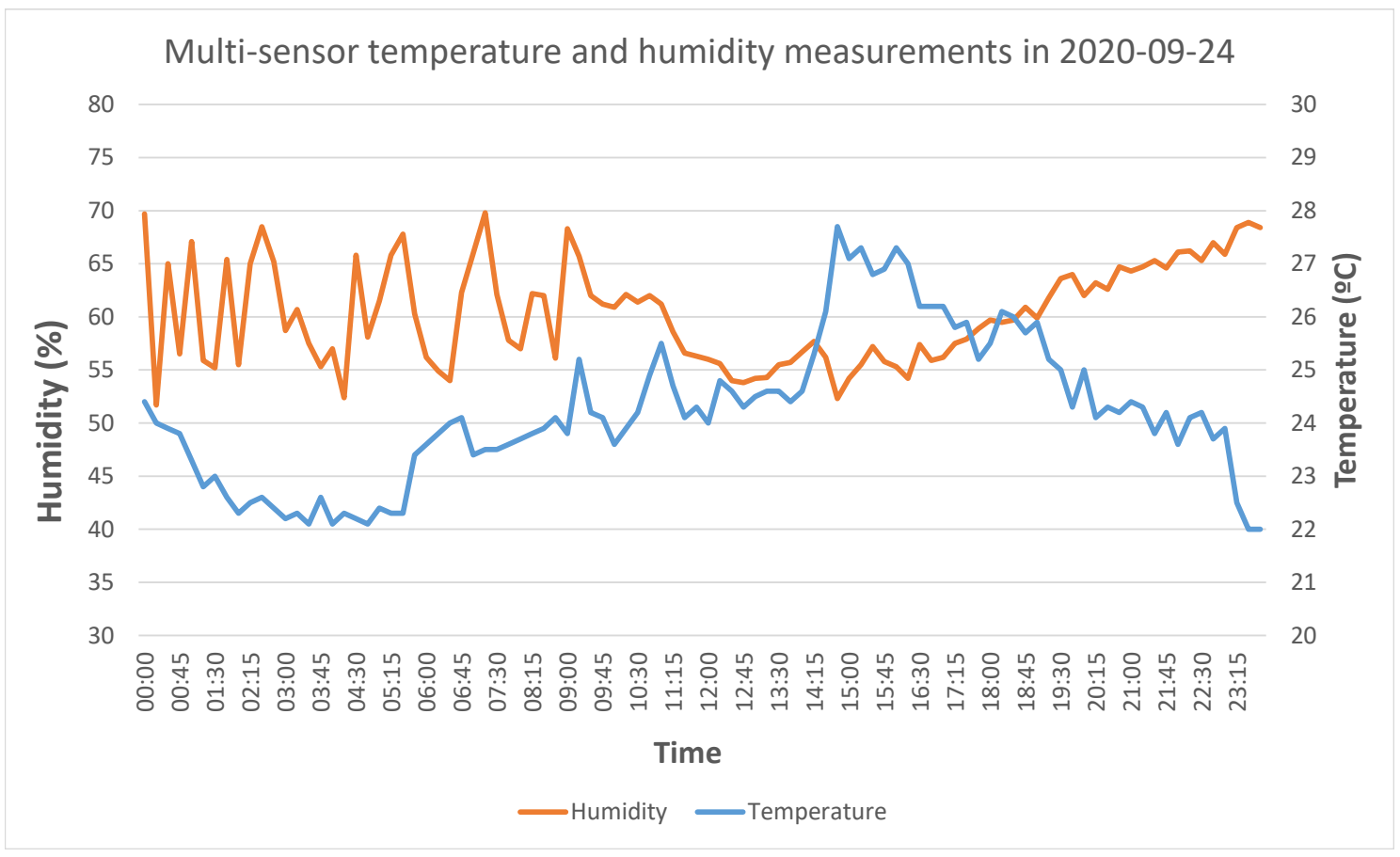

Figure 6. Multisensor measurements for temperature and humidity on 24 September 2020.

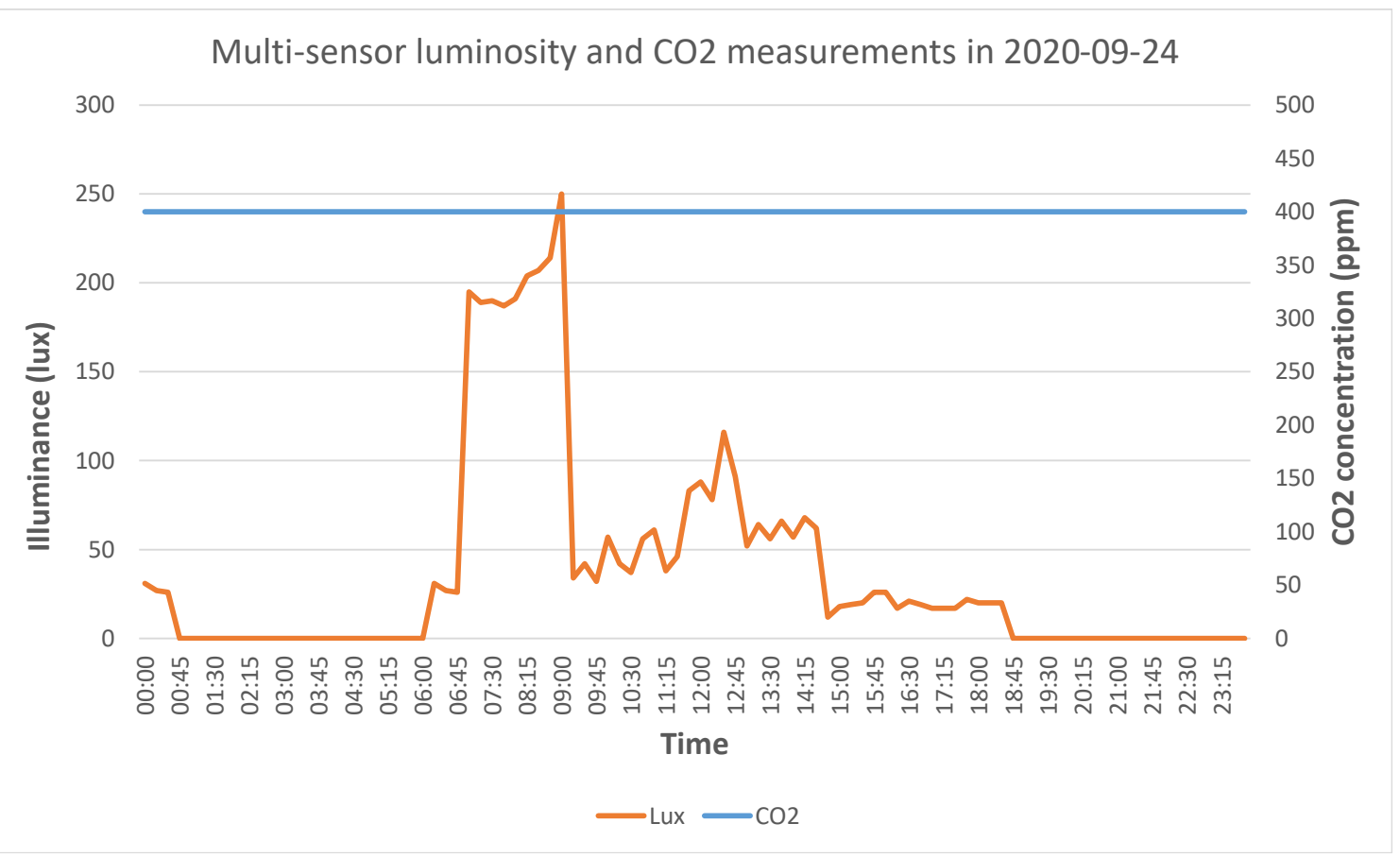

Figure 7. Multisensor measurements for luminosity and $\mathrm{CO}_{2}$ on 24 September 2020.

\section{User Profile Segmentation}

User profiling segmentation in FEEdBACk is based on user behavior, more specifically on the specific motivational drivers and barriers for changing energy behavior that characterize a particular user profile. Pro-environmental values and attitudes were used as the main variable that determines which profile a user is assigned. This section describes our approach to user profile segmentation and how the results are applied in the project.

The three demonstration sites that implement and test the FEEdBACk gamification platform represent three unique settings, most evident in the split between residential 
(Lippe) and nonresidential buildings (Barcelona and Porto). The nonresidential buildings consist of different types of buildings, e.g., a multi-storey office building in Porto and multi-purpose public buildings in Barcelona, which results in a large variation in the types of users as well as how and why they are users in these different buildings. Similarly, the residential buildings represent a range of different types of households. The user profile segmentation task was therefore challenged by accounting for these structural differences while considering how they may interact with the users' energy related behaviors as well as the motivational drivers and barriers for changing that behavior.

The chosen methodology for developing user profiles was to develop an extensive questionnaire that consisted of different sections where pro-environmental values, attitudes, perceptions, and beliefs were explored from different angles. The questions were formulated as statements, which were answered using a 5-point Likert scale, which allowed us to quantify the data sample.

The formulation of questions was based on three models: The Theory of Planned Behaviors (TPB) [11], the New Ecological Paradigm Scale (NEP) [12], and Place Attachment (PA) [13]. The TPB was more recently applied to explain pro-environmental intentions as well as behavior in the workplace [14-16]. As a theory for predicting behavior, the TPB was on one hand cited as being useful $[17,18]$ while other authors highlighted its limitations [19-21].

The NEP scale has been used to measure a given population's environmentally conscious world view [12] and the degree to which an individual feels that they are part of nature rather than separate from it: human activities are seen as directly linked with (and thus affecting) global environmental changes [22]. For an assessment of the application of the NEP scale, see [23].

The notion of place attachment has been used here as a multidimensional construct comprising place dependence, place identity, place affect, and place social bonding [13] and thus as based on both a relationship with the place itself and with the others in that given place. Although primarily used in the context of the natural environment, the place attached is used here in the context of a workplace. We were interested in exploring if-and howthe workplace may become that physical space in which pro-environmental behaviors are valued and may reinforce positive feelings of place attachment, e.g., reinforcing pride, acknowledgement, and satisfaction with the workplace (see also [24]).

The questionnaire can be consulted in Table A2 in Appendix D.

A total of 219 complete responses were collected and analysed. The analytical process had one significant restraint, mainly that the project decided to limit the number of user profiles to six unique profiles. This factor had to be kept in mind when determining how to build the profiles and the associated decision tree. Details about the six profiles can be found in Appendix E. Overall, the analysis had a threefold goal:

- To identify key data (and hence questions) that could be used to develop user profiles. This was done with the purpose of also being able to identify a decision tree for the users' profiling app based on a consolidated version of the user profile questionnaire. The decision tree was used to assign individuals a user profile;

- To define six unique user profiles including a description of the motivational drivers and barriers for each profile;

- To analyze the interrelationship between the motivational drivers and the environmental envelopes of the buildings in the pilots and to segment users within a profile group according to the environmental envelope they are located in.

User segmentation is important as it also is used to ensure that users receive only relevant action-related messages. An example of such a message could be "Remember to open the blinds and use daylight instead of artificial lighting". This message would only be relevant (1) if users can control the blinds and the lights, (2) if they are in a room with windows/blinds, and (3) if windows are situated so that daylight can enter (i.e., Do windows face south, north, east, or west?). Moreover, such a message should also consider the actual real-time weather and time of day. 
In the analysis, we looked for common patterns and trends across the entire data sample, being particularly sensitive to differences between the nonresidential and residential data samples. We also checked the inner consistency of separate sections (e.g., questions representing a unique framework) using Cronbach's Alpha. As a result, the NEP scale was discarded and we focused instead on questions related to the TPB model (notions of place attachment will be explored further in forthcoming work on behavioral change assessment.). When separating the data related to TPB, we found a clear pattern that, even where respondents had similar overall attitudes, values, and beliefs, they had very different perceptions of self-efficacy and how resourceful respondents felt with respect to reducing energy.

Based on the findings from the analysis, a decision tree for user profiling was developed, which allowed the app to automatically assign user profiles based on the combination of 18 questions on pro-environmental attitudes, beliefs, values, and perceptions and 4-6 (demo site specific) segmentation questions. By assigning user profiles to each participant in FEEdBACk, the aim is to stimulate the psychological underpinnings of users' energy behaviors with the purpose of strengthening their intentions and transforming intentions into concrete habits.

Figure 8 shows the decision tree that was be implemented in the users' profiling app to assign each individual player a user profile. It is based on the scores received from the user profiling questionnaire presented in Appendix D.

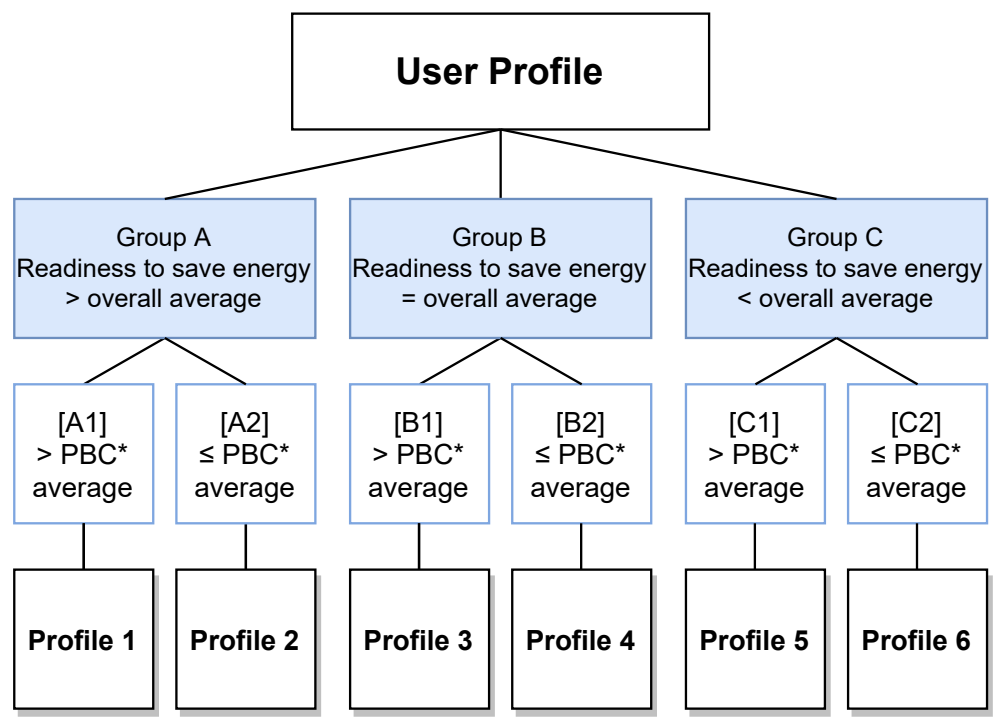

*Perceived behavioural control

Figure 8. User profiling decision tree.

The decision tree was based on the following calculation:

$$
\text { Average }=\frac{x(\text { total score } \div \text { number of questions })}{y(x \div \text { number of entries })}
$$

The above formula must be used to calculate first "Readiness to save energy", which results in three distinctive groups (A, B, and C). Next, the formula is applied for each of these three groups to calculate the "Self-efficacy" score for each. "Readiness to save energy": this is the overall total score based on all 18 questions related to behavior. "Selfefficacy": this is the score based on the 6 questions related to perceived behavior control (PBC) or self-efficacy (see Appendix E). Figure 9 shows an example where the total number of entries (respondents) is 51. 


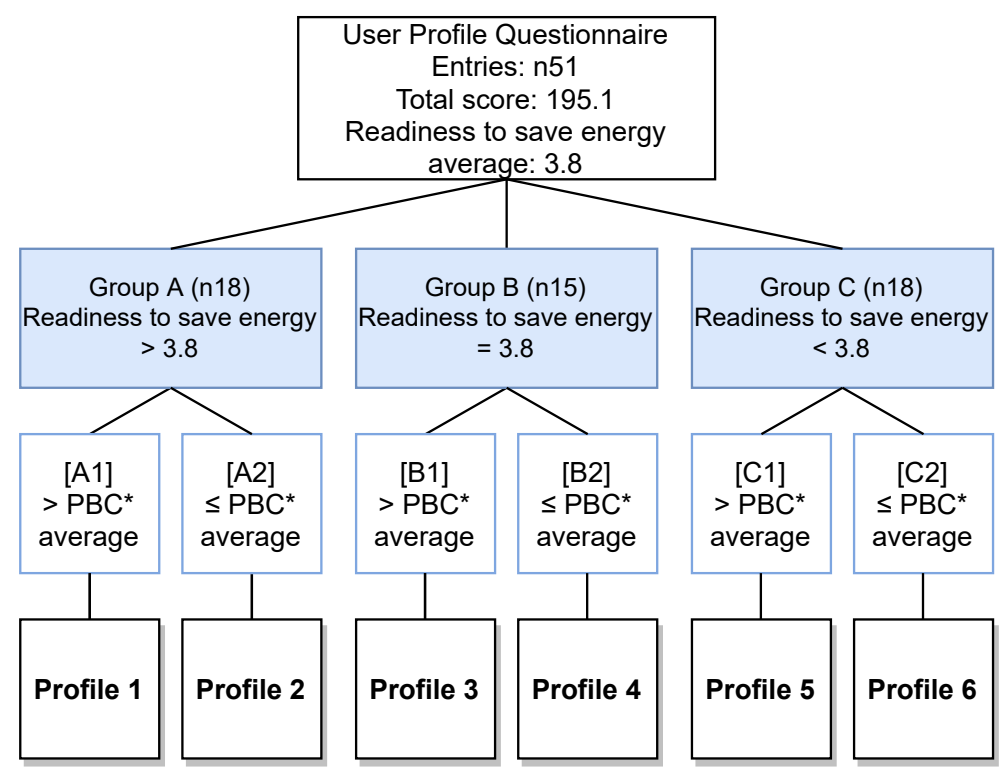

Figure 9. User profiling decision tree using 51 respondents as example.

Figure 10 illustrates the subsequent step, where the focus is on the average score for the six questions related to PBC. In this case, for group A, the average score for PBC is 3.9 and group A was thus split into two groups: Profile A1 and Profile A2. The formula must be applied to groups B and $C$ also, resulting in a total of six unique user profiles, which are presented in detail in Appendix E.

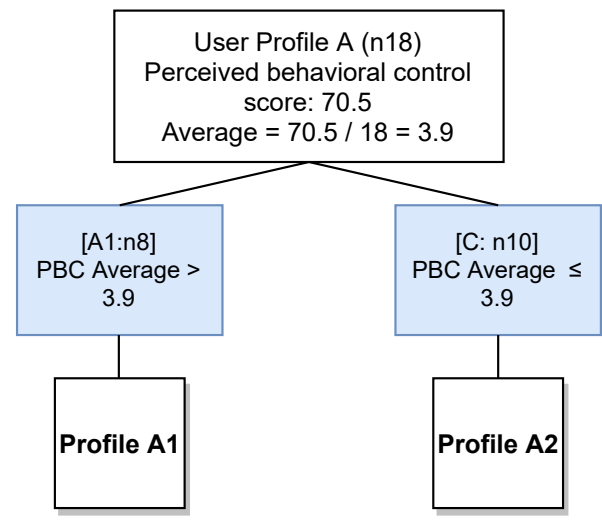

Figure 10. User profiling decision tree applied to respondent number 18.

\section{Gamification Platform}

The gamification platform (Figure 11) consists of a set of elements that aim to provide the user, through a mobile application, with an experience that leads to encouraging a more efficient-energy utilization and a more responsible consumer behavior. The face of this entire ecosystem is the pervasive mobile application (ECOplay), which analyzes context and sends personalized messages through a gamified peer competition. 


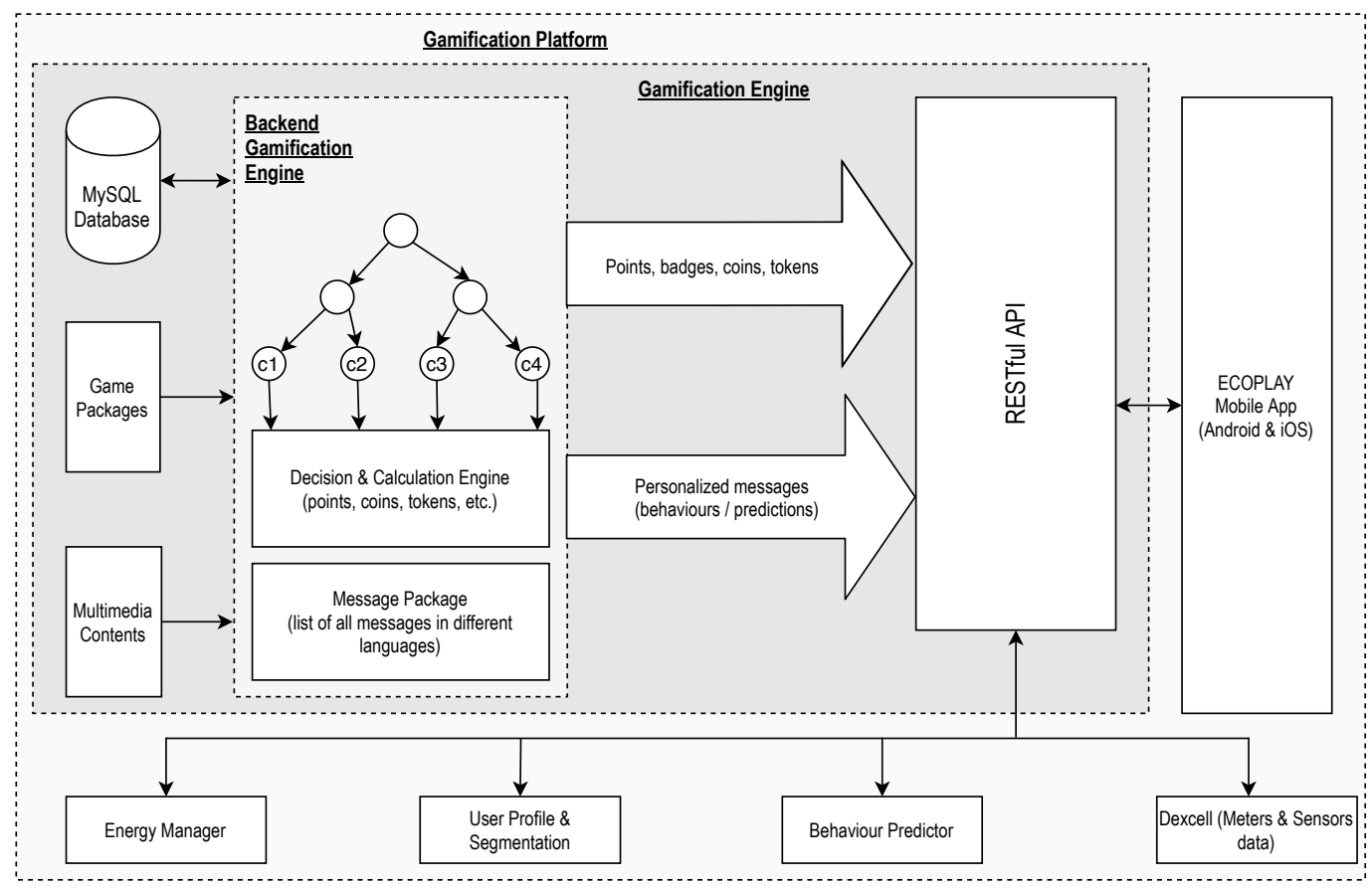

Figure 11. Gamification platform.

As we can see in Section 3.1, the gamification platform engages in a broader interactive energy management system, which aids interested stakeholders in optimizing the utilization of the resources available in individual buildings or blocks of buildings and in maximizing energy savings. This system enables decision support capabilities and interacts with several other applications, such as an automation manager (Section 6.5) and a users' behavior predictor (Section 6.4).

The gamification platform sends several types of messages:

- Alarms: These messages warn the game master when a group is not configured correctly (i.e., a user is no longer working/living on a location). They are displayed on the energy manager Graphical user interface (GUI.)

- Short-term recommendations related to personal environment settings and activity in order to save energy (i.e., please remember to turn off the monitors when you leave the office!). These recommendations are displayed on the gamified mobile app.

- Short-term recommendations related to facility settings and directed to the facility manager (i.e., please turn off the $\mathrm{AC}$ as indoor temperature is below $23^{\circ} \mathrm{C}$ ). These recommendations are displayed on the energy manager GUI.

\section{Mobile Application}

Based on previous research findings (Section 1), a platform based on a gamification approach was developed in the course of the FEEdBACk project, where each user is encouraged to firstly raise their level of understanding of options for energy use and its implications and then to engage them to set their own personal targets, to interact competitively with other users, and to be motivated through different reward schemes according to their group intrinsic characteristics.

The developed mobile application (Figure 12) has an interactive and user-friendly GUI to motivate permanent behavioral change by fostering awareness, understanding of energy options, and consumer engagement through personalized and opportunistic messages and gamified peer competition. 

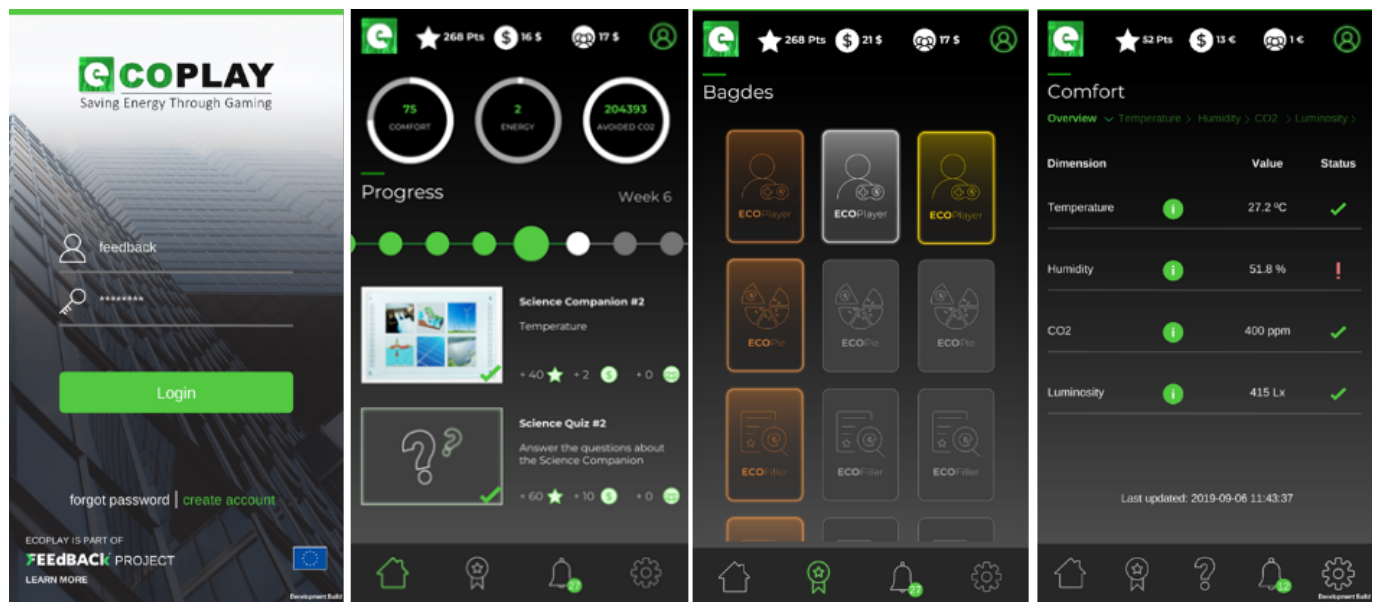

Figure 12. Screens from the mobile application.

The mobile app presents different types of screens, according to the goals set:

1. Login and create accounts for each user, selecting their location;

2. A complete gaming dashboard where the user can understand what is the current progress, what are the next things that must be done, how to obtain scores (individual and team points), and energy- and comfort-related metrics. It is on this screen that they jump to other functionalities;

3. Individual and team leader boards;

4. List of badges with the indication of what was gained and what must be done to earn mores;

5. Comfort measures and graphics;

6. Energy measures and graphics;

7. List of all received notifications;

8. User and application settings;

In addition, the users have the possibility to reflect on their peers' progress towards energy efficiency, thereby increasing visibility, awareness, and commitment. Levels of learning and understanding energy use also is provided by videos, quizzes, and other learning objects that are available weekly according to a learning and engagement plan.

The developed gamification engine does not have to simulate or model the potential behavior change-it will be able to aggregate the data from media-enabled training programmes plus anecdotal evidence through user storytelling and then actually measuring the impacts, both technical and emotional, on a daily basis. This allows for significantly improving the range and quality of information and behavioral understanding.

\section{New ICT and Data Analysis Applications Developed in FEEdBACk}

\subsection{Net Load Forecasting and Baseline}

The Net Load Forecasting and Load Baseline application is composed by three core services:

1. Photovoltaics (PV) Forecasting Service generates photovoltaic energy generation forecasts, with 15 min time resolution, for a time-horizon up to $72 \mathrm{~h}$ ahead. It was applied for the case of INESC TEC (see Appendix B), for the solar panels installed in the building rooftop, which amounts to $16.38 \mathrm{~kW}$ of installed power;

2. Load Forecasting Service generates energy consumption forecasts, with $15 \mathrm{~min}$ time resolution, for a time-horizon up to $48 \mathrm{~h}$ ahead. This forecast is trained to take into account the most recent available data;

3. Load Baseline Service provides daily estimations of power consumption profiles for the preceding day, with $15 \mathrm{~min}$ time resolution. This forecast is based on historical measurements of data prior to the actions promoted in the scope of the project. 
Combining objectives 1 and 2 allows for obtaining the building net load forecast, which is of interest for maximizing the building self-consumption. Combining objectives 2 and 3 allows for quantification of the behavioral change induced by the project.

The structure of the application and respective services is illustrated in Figure 13.

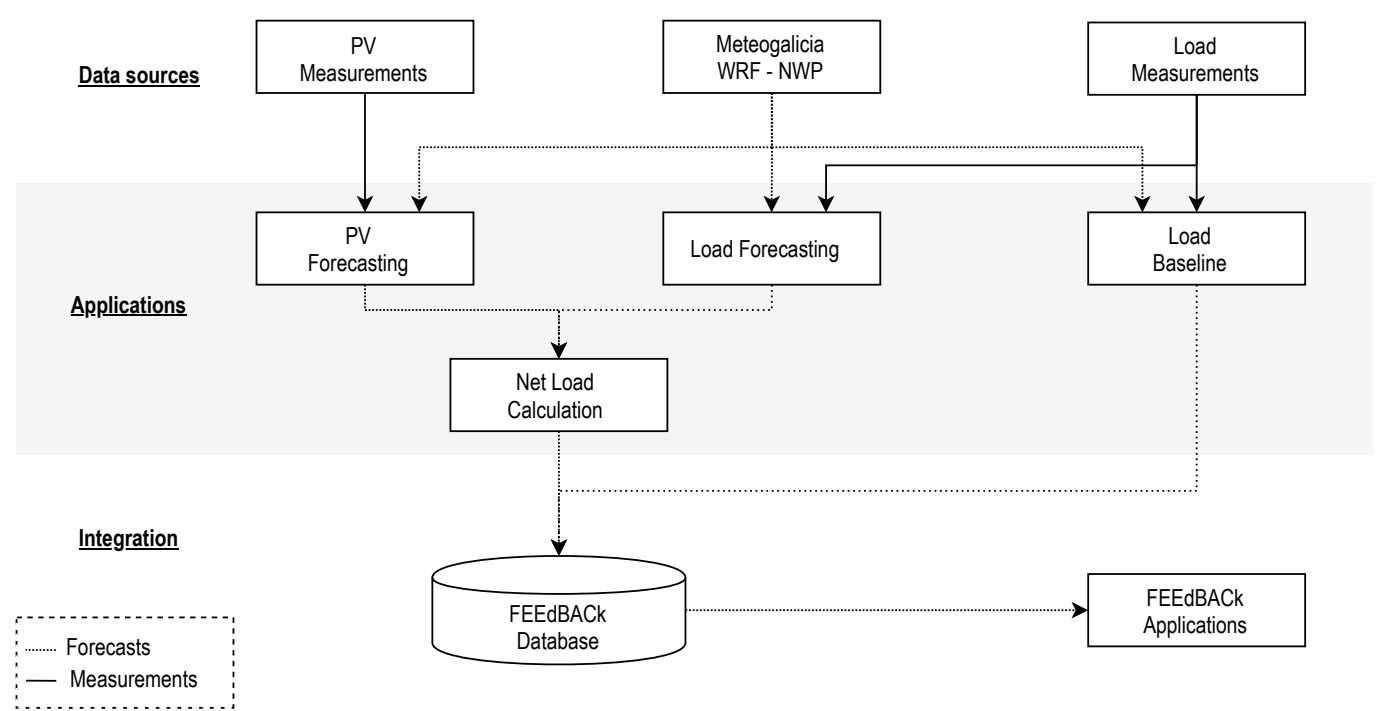

Figure 13. Block scheme of the net load and load baseline forecasting applications.

The services presented in Figure 13 are constituted by supervised machine learning models that exploit exogenous information such as numerical weather predictions (NWP) combined with on-site measurements data (PV energy generation and energy consumption gathered by FEEdBACk project data metering systems) to produce the respective outputs (e.g., forecasts or baseline estimations).

The historical and daily NWP data used by all the services in this application were generated by the mesoscale Weather Research and Forecasting (WRF) model. This information is currently available at MeteoGalicia THREDDS server (https: / /www.meteogalicia.gal/ web/modelos / threddsIndex.action (accessed on 15 February 2021)), which is a publicly available service that enables access to data catalogs of multiple data providers. For each service (e.g., PV forecast, load forecast, and load baseline), a selection of weather variables is explored.

\subsubsection{Load Forecasting}

The Load Forecasting Service is responsible for providing short-term forecasts on energy consumption for different appliances (such as lights, outlets, and HVAC, among the others mentioned in Section 3.2). It consists of a framework of various forecasting models, combined to provide point forecasts up to a $48 \mathrm{~h}$ ahead of the time horizon. This ensemble aims to leverage the performance of each individual model, thereby mutually improving their quality and producing better forecasts.

The models, in general, depend on on-site historical energy consumption measurements data and exogenous information such as WRF-NWP variables (which are described in Table 2).

Table 2. Load forecasting model numerical weather prediction (NWP) input variables

\begin{tabular}{ccc}
\hline Variable & Units & Description \\
\hline temp & $\mathrm{C}$ & Ambient temperature at $2 \mathrm{~m}$ \\
$\mathrm{rh}$ & $(0,1)$ & Relative humidity at $2 \mathrm{~m}$ \\
\hline
\end{tabular}


The process is presented in Figure 14. After accessing the necessary data, feature engineering techniques are used to create new variables that improve the forecasting quality of the models. Such a set of features includes calendar variables, lags of the historical measurements, and a selection of weather variables (and/or their lagged variants). An analysis of the days subject to forecasting determines the presence of holidays or bridge days, which influence the subset of historical data used as input and for which features are selected for the models. In the case of regular days, an extra selection step, based on correlation, defines the subset of features used as input. The requisites are as follows:

- Naive model: only the last equivalent day consumption records are needed.

- Model based on data from last week (LastWeek) : for this model, only calendar variables are used to select previous seven days' same-hour records and prediction is a weighted average of those values.

- $\quad$ Conditional Kernel Density Estimation (cKDE) and Gradient Boosting Trees (GBT): a subset of all the engineered variables as mentioned above are fed into these models.

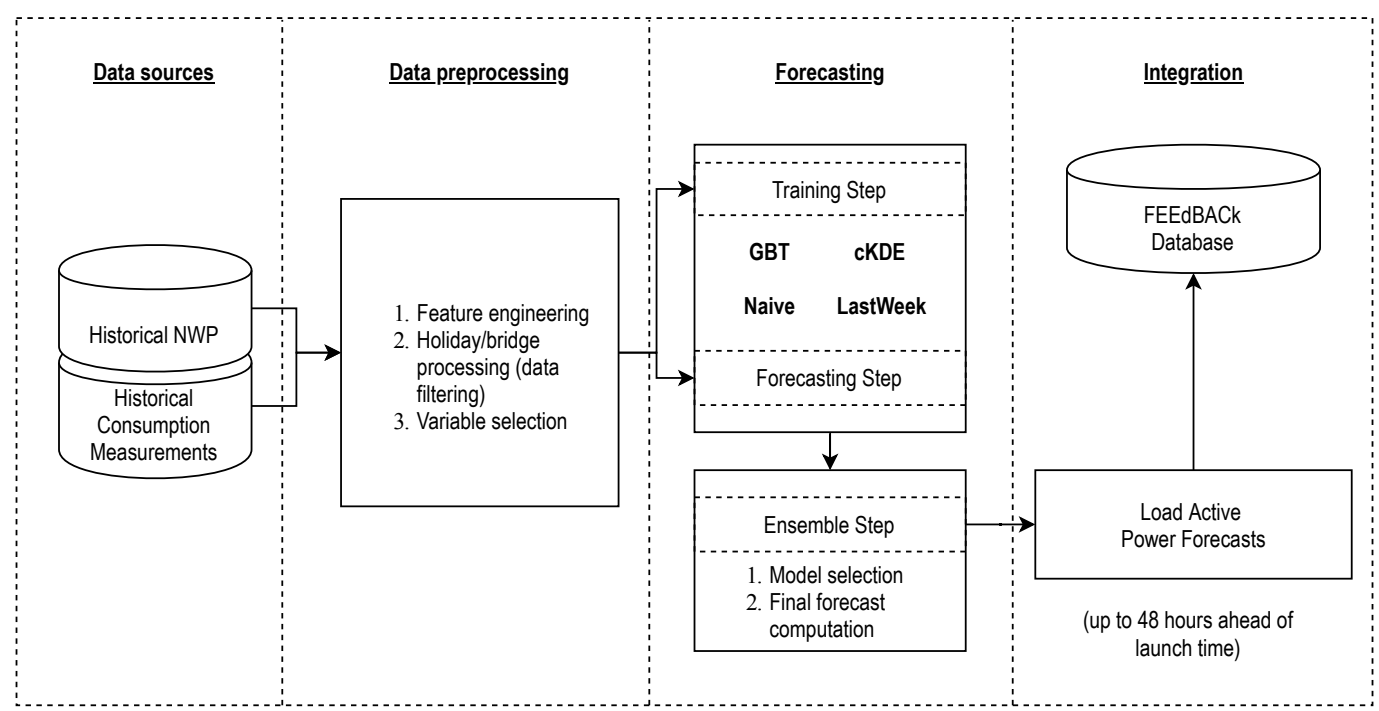

Figure 14. Block-scheme of the load forecasting application.

For GBT regression, two different implementations are used, from the Python package sklearn [25] and the framework provided in LightGBM [26]. In the case of cKDE [27], online optimization of hyperparameters using the Nelder-Mead algorithm is also performed in order to improve the forecast quality. A total of five different models are applied to each forecast computation. In the ensemble phase, their performance is evaluated using last month's available forecasts and the two best models are selected, after which the mean value of those two models' forecasts is used as the final output. Not all five models are used in order to avoid averaging with models that recently performed worse. Finally, the results are stored in the database to be used for calculation of the net load forecast (along with the PV power forecasting module output from the next subsection) and other applications. Table 3 shows the duration of a single run of the service considering the various phases of the process. 
Table 3. Computational times for a load forecast run considering one year of historical data.

\begin{tabular}{cc}
\hline Task & Computation Times (Seconds) \\
\hline Data Processing & 1.99 \\
Naive model & 0.16 \\
Last week's data model & 0.05 \\
cKDE & 0.53 \\
GBT & 6.77 \\
LightGBM & 0.76 \\
Ensemble & 0.42 \\
Total & 10.68 \\
\hline
\end{tabular}

\subsubsection{PV Power Forecasting}

The PV power forecasting service relies on machine learning models combined with feature engineering techniques to generate short-term (up to $72 \mathrm{~h}$ ahead) solar energy forecasts. These forecasts are later used by the Net Load Forecasting Application to estimate the future net load of the INESC TEC building.

The forecasting models embedded in this application depend on the following data sources: (1) historical power production measurements from local power plants (e.g., household or buildings PV panels) and (2) numerical weather predictions (NWP) for a predefined selection of weather variables.

Analogous to the Section 6.1.1 application, the NWP variables are extracted on a daily basis from the Meteogalicia THREDDs server. The selection of weather variables for this service is presented in Table 4.

Table 4. PV forecasting model NWP input variables.

\begin{tabular}{ccc}
\hline Variable & Units & Description \\
\hline swflx & $\mathrm{W} / \mathrm{m}^{2}$ & Surface downwelling shortwave flux \\
temp & $\mathrm{K}$ & Ambient temperature at $2 \mathrm{~m}$ \\
$\mathrm{cfl}$ & $(0,1)$ & Cloud cover at low levels \\
$\mathrm{cfm}$ & $(0,1)$ & Cloud cover at mid levels \\
cfh & $(0,1)$ & Cloud cover at high levels \\
cft & $(0,1)$ & Cloud cover at low and mid levels \\
\hline
\end{tabular}

The machine learning pipeline considered in application is illustrated in Figure 15.

As depicted by Figure 15, there are four steps in the data pipeline. Initially, NWP and raw energy generation measurement data were retrieved from the FEEdBACk project central database. These timeseries were then temporally aligned and imputation techniques (i.e., linear interpolation) were used to replace the missing values in between hours (no imputation was performed for more than one hour of missing values).

To improve the quality of forecasts, feature engineering techniques were applied individually to each raw NWP timeseries. The techniques implemented for this application result from the extensive study in [28]; therefore, only a brief description of each is provided below.

- $\quad$ Temporal variance $\left(\sigma_{\text {time }}^{2}\right)$ : Three temporal variance indicators considering $N_{h} \in\{3,7,11\}$ hours moving windows centered in a specific lead-time of interest $(t+k)$ :

$$
\sigma_{\text {time }}^{2}(t+k)=\frac{\sum_{i=t+k-N_{h}}^{t+k+N_{h}}(x(i)--\bar{x})^{2}}{N_{h}-1}
$$


- $\quad$ Average of past NWP runs ( $\bar{x}_{\text {past }}$ : average of the four available forecast runs $\left(x_{d}\right)$ for a specific lead-time of interest $(t+k)$.

$$
\bar{x}_{\text {past }}(t+k)=\frac{\sum_{d=0}^{3} x_{d}(t+k)}{4}
$$

- $\quad$ Lags and leads $(t+k \pm z)$ : Preceding (lags, $t+k---z, z=1,2, \ldots$ ) and following (leads, $t+k+z, z=1,2, \ldots)$ with respect to each lead-time $(t+k)$ in the forecast horizon.

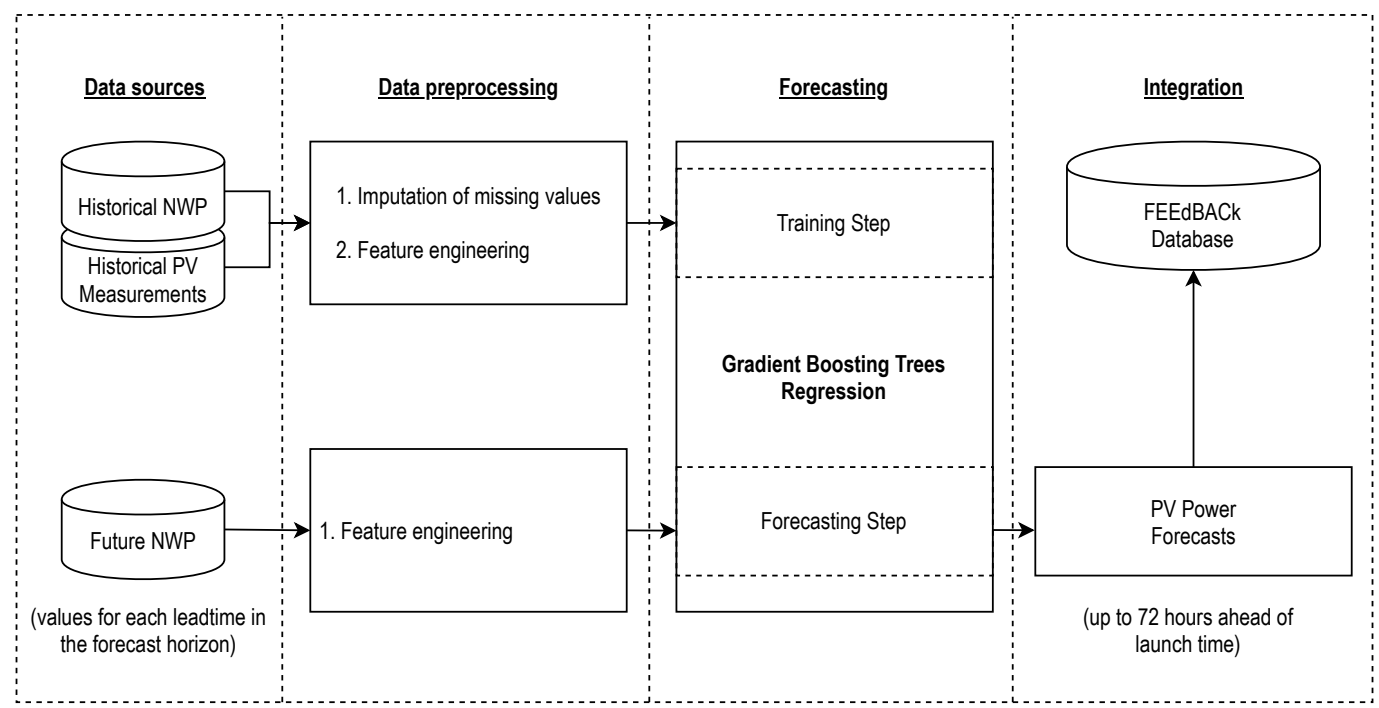

Figure 15. Block-scheme of the pv forecasting application

The combination of feature engineering techniques with nonparametric models such as gradient boosting trees (GBT) was effective regarding solar power forecasting. As an example, the top performing models in the Global Energy Forecasting Competition 2014 (GEFCom2014) included gradient boosting forecasting models in their methodology. Additionally, the model that ranked second only used on-site information, which means that great improvements in the forecast quality can be obtained by carefully exploring local information $[29,30]$.

In this work, the Python open-source scikit-learn library [25] GBT implementation was used. The models were trained on a supervised fashion (i.e., weather-to-power model) considering the historical up-to-date PV measurements and NWP-WRF data. To maximize the forecasting quality, the GBT hyperparameters were optimized using the Bayesian optimization algorithm [31] using the package [32].

The trained models were then used to produce PV power forecasts using daily weather forecasts as input information. These forecasts were then stored in the FEEdBACk project central database to be used later by the remaining software applications (e.g., Net Load Forecasting Application).

Considering that the entire framework illustrated in Figure 15 runs upon requests and its forecasts are subsequently used to calculate net load forecasts, it is important to assess the total computational time of each forecast run. Table 5 presents the computational times of every step in the forecast pipeline. The results were extracted from a single run on a Intel(R) Xeon(R) CPU E3-1245 v5 @ 3.50 GHz with 32 Gb RAM. 
Table 5. Computational times for a PV forecast run considering one year of historical data.

\begin{tabular}{cc}
\hline Task & Computation Times (Seconds) \\
\hline Data Processing & 0.33 \\
Train & 5.90 \\
Forecast & 0.05 \\
Total & 6.28 \\
\hline
\end{tabular}

\subsubsection{Load Baseline}

The baseline estimation component consists on forecasting energy consumption based on an alternative scenario where there are no control actions. In this case, we understand that these actions will be stimulus given to end users in order to influence their own consumption behavior and consequently taking control over load curves. Therefore, the deployed statistical models are based on consumption patterns before the implementation of the FEEdBACk project. The data points are produced as output displayed in the form of time series. These values correspond to consumption granules of $15 \mathrm{~min}$ serving as a comparison to real equal-resolution consumption metering (as in Section 3.2). Thus, we can calculate energy savings when we differentiate that variable pair. In order to develop statistical models for estimation of (what we designate by) load baseline along the time, we need predictors such as simple calendar factors (hour, day of the week, month, etc.) and exogenous variables to tackle a multiple regression problem.

The seasonal variables are inferred from timestamps collected in energy consumption databases. For the FEEdBACk project, we obtained meteorological conditions (irradiance, temperature, relative humidity, and cloudiness) as exogenous predictors. Measures of these explanatory variables are registered in the three demo municipalities. MeteoGalicia (https: / / www.meteogalicia.gal/web/modelos/threddsIndex.action (accessed on 15 February 2021)) service provides datasets with that type of one-hour granularity measurement joined to each consumption node dataset through interpolation, with municipality in common.

Regarding the creation of most effective predictive models, we took feature engineering and parameter tuning as a starting point. Gradient boosting trees (GBT) [25] was chosen as a regression algorithm for application because (I) it performed better for an instrumental variable estimation, (II) it has large flexibility in parameter optimization, and (III) it does not need scale transformation at the level of predictors. As scoring criteria, we adopted the mean absolute error divided by maximum history consumption measure verified on each node.

Firstly, the tested models were oriented for distinct predefined feature subsets, where it distinguished them from several weather conditions. For a first collection of consumption nodes integrated at the Porto demo site, models combining four variable subsets with two temporal resolutions and using fixed parameter settings were tested. Therefrom, several node models sharing the best scores up to third decimal place were checked. Even so, most results kept the score below the naïve model (estimations based on previous-day consumption).

Due to unsatisfactory results, feature extraction after was adopted, namely indicator variables and others such as season of the year or monthly minimum/maximum average consumption. About weather features, only the (external) temperature predictor was kept because it correlated significantly better with load consumption (than the remaining ones) in most consumption nodes. Instead of feature-oriented modeling to achieve greater assertiveness on statistical models, parameter optimization was used on the GBT algorithm through Grid Search using the 9-fold cross validation [25] method. Therefore, the closest results to real consumption where nodes with regularity were achieved.

Thus, a forecasting model suitable for each consumption node was produced, meaning assignment of realistic estimations to distinct devices, rooms, floors, buildings, and demonstrators. Considering a specific parameter tuning per node, an operational module (see Figure 16) was developed, capable of estimating the baseline for a certain time horizon 
and taking into account launch time (beyond system time). If estimation day is ordinary (neither a public holiday nor bridge), then the statistical model applies predictions based on timestamps inferred from the forecasting window. This type of model only accepts energy forecasts if temperatures are completely available.

In the case of problems related to weather info database, alternative models are used, which were trained with no exogenous variables. For holiday scenarios, a rule scheme is followed consisting of previous-consumption simple averages by each time of day corresponding to the same times according to the applied rule. Output values are sent to the respective demo database, where datasets contain timestamps with node addresses, request times, measured units, and energy forecasts.

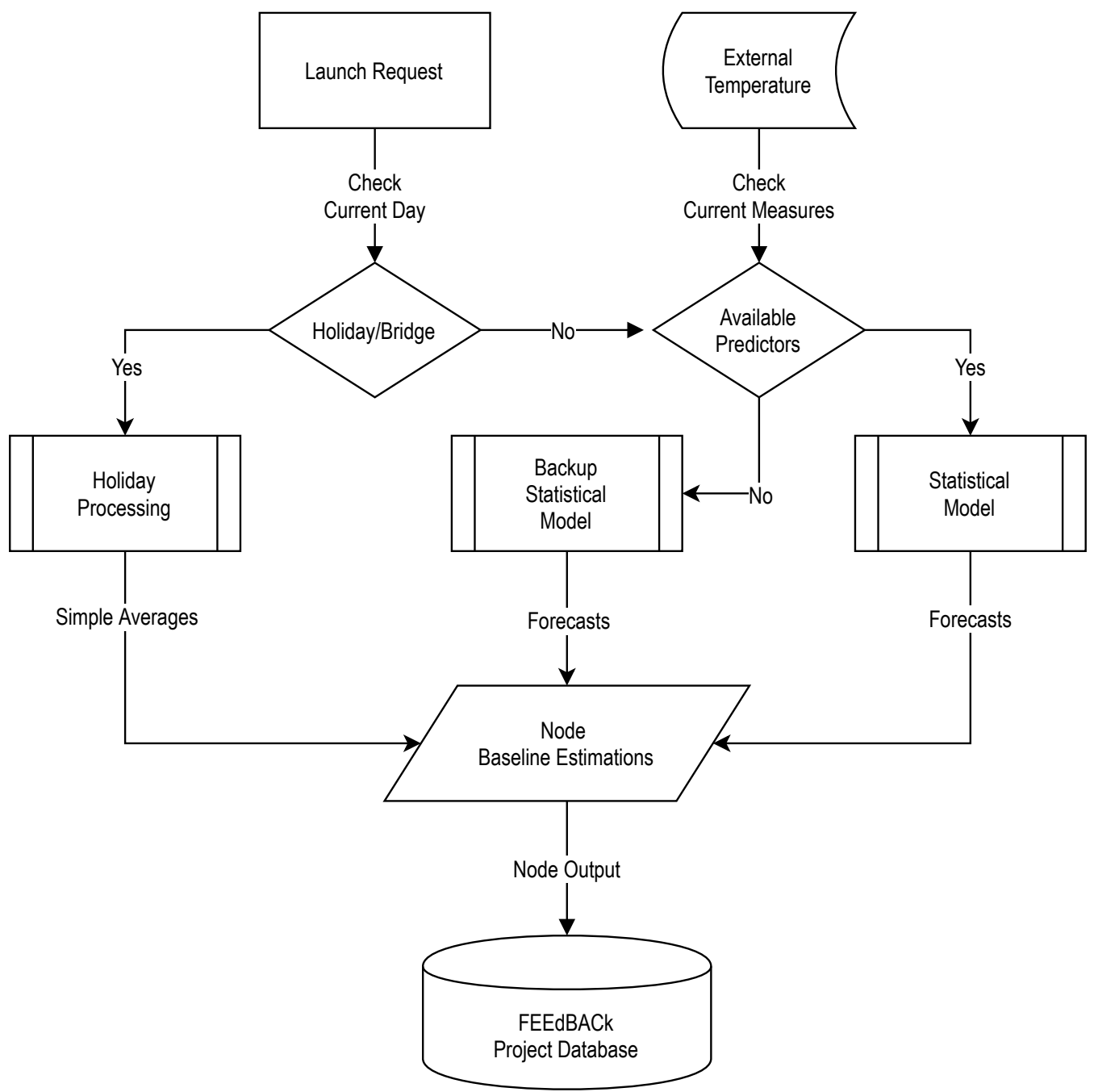

Figure 16. Block scheme of the load baseline estimation service.

\subsection{Load Disaggregation}

Load disaggregation application aims to provide an overview of the electricity consumption in buildings. For instance, it transforms the whole-building aggregated power measurements to specific consumption values allocated to categories of devices. The application allows us to avoid physical monitoring of individual appliances' power consumption employing nonintrusive load monitoring (NILM) [33]. Therefore, such an approach not only contributes to preserving occupants' privacy but also saves effort and cost of sensors installation at each power outlet in the building. Moreover, load disaggregation techniques are suitable for both tertiary and residential buildings. NILM methods can be classified into various groups, such as supervised and unsupervised [34], and low-frequency and 
high-frequency methods. The latter frequency-based division is determined in detail in the works of $[35,36]$. The load disaggregation approach deployed in the current project falls into the unsupervised low-frequency category and is thus suitable for disaggregating power measurements obtained from conventional smart meter at a 15-min resolution.

Figure 17 represents the application's block scheme, where the primary input and output data streams are depicted. The unsupervised device usage estimation (DUE) algorithm [37] represents the core of the load disaggregation application for residential households. The DUE differs from the methods proposed previously in the literature by being a hybrid methodology between load profile simulation and load disaggregation. Due to its unsupervised nature, the algorithm does not require any training. Therefore, it extracts essential model parameters from various available data sources.

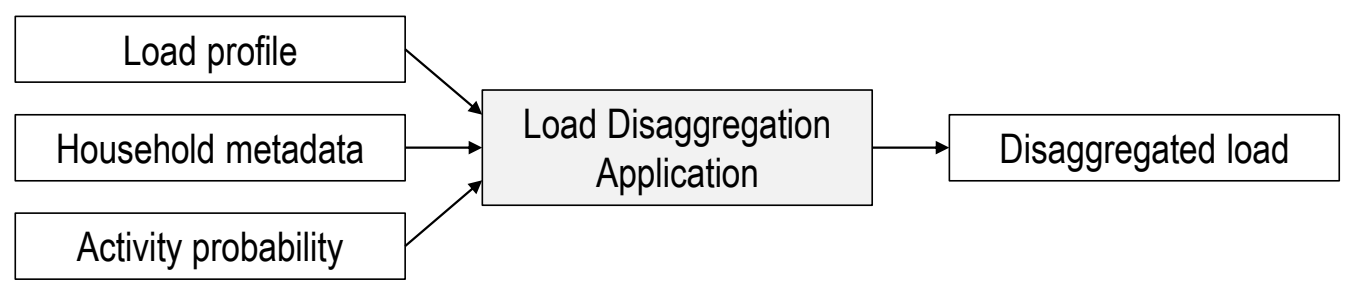

Figure 17. Block scheme of the load disaggregation application.

There are three types of inputs required for the DUE algorithm. First, the load profile of the household is obtained, which consists of aggregated low-frequency active power measurements at 15-min resolution. Second, household metadata is collected using targeted or publicly available surveys. The essential information includes the list of appliances present in the house; the characteristics of the inhabitants, namely number, age group, and employment status; and the frequency of using various devices. Third, probability statistics of daily activities are plugged in from the time-of-use survey. The algorithm requires that these data generate activity chains for each member of the household based on the Markov model. On the output side, the DUE algorithm provides disaggregated load curves according to 8 distinct categories: cooking, entertainment, fridge, heating, information and communication technology, standby, housekeeping, and light. Each category is composed of various common household appliances and the interested reader can refer to [37] for more details.

The workflow of the DUE algorithm can be described in two steps: pretreatment and the main disaggregation procedure. As the methodology is sequential, it has to be repeated for each of the days of interest in the household load curve. First, the standby and the fridge consumption are filtered out from the power signal. The prior is identified as the minimum power level of the considered day; thus, occasionally, it includes the constant part of the fridge consumption. The latter is extracted from the night time periodic signal of all the days in consideration and is further synchronized with the power signal of each particular day. Once these two categories are detected in the load curve, they are subtracted from the power measurements and the algorithm proceeds to building activity chains for each inhabitant above the age of 10 . The methodology varies between inferring the activity chain for a teenager and an adult. Activities are allocated with respect to the occupancy patterns of the household, which can be deduced from the power peaks of the load curve. As partial occupancy is not considered, all inhabitants are assumed to be present in the house when a power peak is detected. Teenagers between the ages of 10 and 18 are expected to have unpredictable activity chains; therefore, they are treated separately and their chains are created randomly. Once activity chains are generated for each member of the household, the algorithm allocates respective appliances based on their usage probability per activity, usage duration, and their power demand. The optimization procedure described is repeated until the difference between the measured and the simulated load curves is smaller than the predefined tolerance. 
Despite being unsupervised, the DUE algorithm delivers estimation accuracy comparable to one from the supervised NILM algorithms. Moreover, computational performance is the advantage of the DUE algorithm. Due to its sequential nature, the execution time scales linearly with the length of the dataset. Therefore, the user of the load disaggregation application can choose historical periods of variable duration to overview their electricity consumption patterns.

\subsection{Occupancy Forecast}

The occupancy forecasting application supports other applications by indicating a building's occupancy for the days ahead. Depending on the demonstration site, the app provides forecasted presence and absence information by sub-metering zone, by functional area, or by a house as a whole. The algorithms to forecast occupancy can be classified into schedule-based and context-aware [38]. The latter approach, at the core of the developed application, depends entirely on sensing the conditions of indoor climate or by approximating it through electricity or water usage. The methodology is based on indirect occupancy detection; hence, no devices of which the purpose is precisely to detect the presence, such as video cameras, passive infrared sensors, or motion sensors, are used. The choice of an indirect approach was made for two reasons. First, it preserves users' privacy as the occupancy information is inferred contrary to being collected. Second, it allows us to effectively exploit meters and sensors already in place or installed in the course of the project. Another way to classify the occupancy algorithms is to divide them into supervised and unsupervised, where some of the most prominent works in the field [39,40] deploy the prior and the latter methods, respectively.

There are two types of algorithms developed for occupancy forecasting in the framework of the FEEdBACk project, with their methodology, design, validation, and testing detailed extensively in [41]. The general algorithm is fully unsupervised, and it can be applied to all demonstration sites. In the particular case of the Porto demonstration site, a supervised version of the algorithm can be used instead of an unsupervised one. It takes the advantage of inferring day-ahead occupancy from electrical consumption data and historical occupancy information available in Porto. In both cases, the output of the algorithm is similar: binary vector of occupancy, indicating presence or absence, with a defined time resolution.

Figure 18 describes the supervised occupancy forecasting algorithm. The necessary input information contains both historical and forecasted data streams. In order to enable the algorithm's learning, historical load profile and occupancy patterns need to be collected. The prior constitutes low-frequency electrical consumption measurements, which can be obtained from a conventional smart meter. The latter is represented by clock point occupancy recorded at building entrances. The forecasted load profile is constructed using the methodology described in Section 6.1.

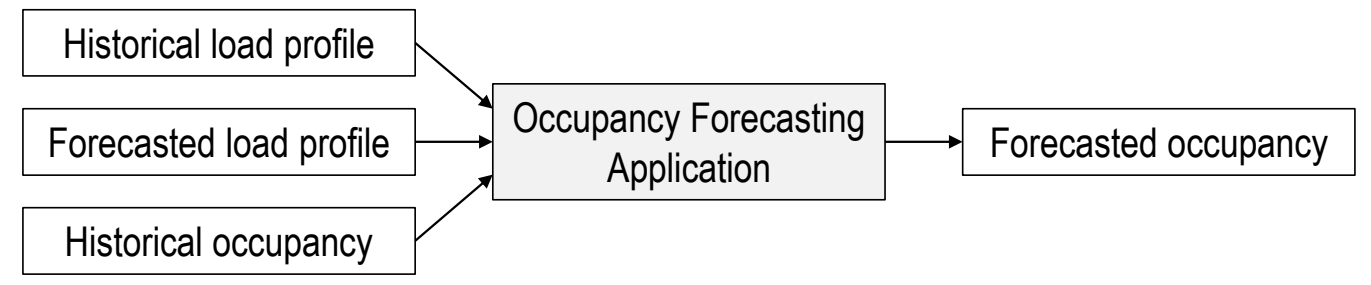

Figure 18. Block scheme of the occupancy forecasting application based on supervised method.

The main process steps include preprocessing and modelling. First, the initial data are loaded. To improve its quality, any erroneous measurements are removed and missing values are imputed. Second, the raw data are transformed into meaningful data sets using feature extraction. In this work, the novel method of extensive feature engineering is proposed. Over 60 manually designed features are segmented into three main categories: statistical features, load curve shape features, and time-related features. Third, feature 
scaling and standardisation are applied to avoid uneven influence of magnitudes on the algorithm's learning. The last step before modelling includes feature selection based on principal component analysis and recursive feature elimination combination, as not all the features contribute equally to occupancy inference. In the modelling phase, the support vector machine is deployed to produce day-ahead occupancy forecasts.

Figure 19 depicts the block scheme of the unsupervised occupancy forecasting algorithm. The method relies on ambient environment data to estimate occupancy. Such data include measurements of relative humidity, indoor temperature, $\mathrm{CO}_{2}$ concentration, and luminosity and can be obtained using the multisensor solution developed in Section 3.3.

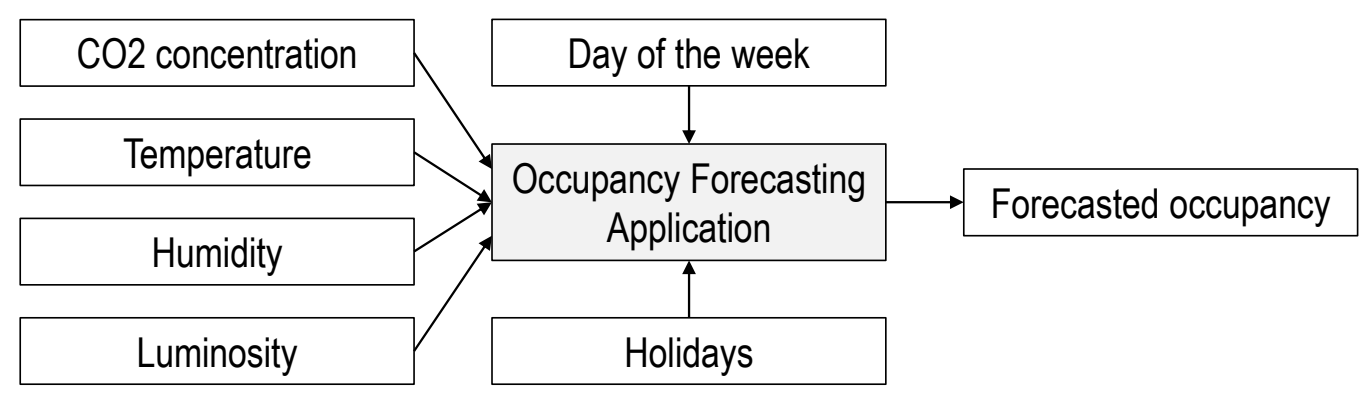

Figure 19. Block scheme of the occupancy forecasting application based on the unsupervised method

The algorithm works by executing two parts sequentially. First, the occupancy is detected from historical ambient environment measurements using unsupervised machine learning techniques: K-means clustering and majority voting. Second, powerful time series forecasting models are deployed to produce day-ahead occupancy from occupancy detected in the historical period. Examples of such models include long short-term memory neural network and Prophet [42]. Additional regressors such as day of the week, time of day, and national holidays can be applied to improve the forecasting accuracy.

For both algorithms, the output data can be represented as the binary occupancy vector with 15-min resolution. Therefore, the day-ahead forecast consists of 96 values, where 1 indicates presence and 0 indicates absence. In case a higher level of detail is required, the same algorithms can be utilized to produce forecasts related to the number of people or the occupation level in a particular space, ranging from low to high. Both supervised and unsupervised occupancy forecasting algorithms were extensively tested on the dataset collected from the Porto demonstration site and showed the highest prediction accuracies of $98.3 \%$ and $97.6 \%$, respectively [41].

\subsection{Behavior Predictor}

The behavior predictor resorts to machine learning techniques and serves the purpose of identifying opportunities for lowering electricity consumption, departing from the overarching objectives of feedback, that is, more than the act of saving energy, the main goal is inducing behavioral change that will save energy. In a resumed way, the output of the behavior predictor is a set of alert messages that users receive in the mobile app (Section 5) that coincide with periods of time where consumption is forecasted as "excessive", although not intended to interfere with the users' comfort levels.

The objectives of the behavior predictor are threefold:

- Identify opportunities for behavioral change to be sent to users in the form of smartphone alerts;

- $\quad$ Predict the impact of such alerts, regarding energy savings;

- Keep track of the evolution of behavior reactions to the sent messages.

The behavior predictor is in fact constituted by 5 predictors, each addressing different issues (see Table 6 for more details) and is intended to be applied for each team according to specifications of Section 4. As a result, there are 75 models for behavior prediction at the INESC TEC building in Porto ( 5 models $\times 15$ teams $=75$ models). 
Table 6. Behavior Predictor models specification.

\begin{tabular}{|c|c|c|c|}
\hline Model & Objective & $\begin{array}{l}\text { Condition for Message to Be } \\
\text { Sent in Real-Time Stage }\end{array}$ & Example of Message \\
\hline plugs empty & $\begin{array}{l}\text { detect excessive plugs } \\
\text { consumption for empty room }\end{array}$ & room is occupied & $\begin{array}{l}\text { "Your workday is almost over. } \\
\text { By unplugging all devices, your team can } \\
\text { save up to } 1.2 \text { KWh per team member, } \\
\text { corresponding to an annual saving of } 20 \\
\text { euros per individual. Is your team ready?" } \\
\text { (for profile 1) }\end{array}$ \\
\hline plugs occupied & $\begin{array}{l}\text { detect excessive plugs } \\
\text { consumption even for } \\
\text { occupied room }\end{array}$ & room is occupied & $\begin{array}{l}\text { "Attention: a great moment is coming. In the } \\
\text { next hours the energy will be more precious. } \\
\text { Please make sure your team has plugged in } \\
\text { only what it really needs. Change (and win } \\
\text { rewards) is in your hands" (for profile 2) }\end{array}$ \\
\hline lights & $\begin{array}{l}\text { detective excessive lights } \\
\text { consumption for empty room }\end{array}$ & room is occupied & $\begin{array}{l}\text { "«I want you to panic and act as if the house } \\
\text { is on fire. Because it is» (Greta Thunberg). Be } \\
\text { the hero and turn off the lights before leaving } \\
\text { your workplace." (for profile } 3 \text { ) }\end{array}$ \\
\hline hvac moderate & $\begin{array}{l}\text { detect excessive heating or } \\
\text { cooling consumption taking into } \\
\text { account that outdoors } \\
\text { temperature is moderate for } \\
\text { the season }\end{array}$ & $\begin{array}{l}\text { room is occupied, actual } \\
\text { temperature outdoors matches } \\
\text { the forecast and is still } \\
\text { "moderate" for the season }\end{array}$ & $\begin{array}{l}\text { "Aren't you sure about what you can do for } \\
\text { the environment? We have detected an } \\
\text { opportunity that will help you: there is a } \\
\text { moderate temperature outdoors (less than } \\
26^{\circ} \mathrm{C} \text { ). Why do not you turn off the air } \\
\text { conditioner and open the windows?" (for } \\
\text { profile } 4 \text { ) }\end{array}$ \\
\hline hvac empty & $\begin{array}{l}\text { detect excessive hvac } \\
\text { consumption for empty room }\end{array}$ & room is occupied & $\begin{array}{l}\text { "Have you been creating value for INESC } \\
\text { TEC since the beginning of the day? Keep on } \\
\text { doing that even at the time you leave! The } \\
\text { room is expected to be empty in the next } \\
\text { hours so make sure the air conditioning is } \\
\text { turned off at night. INESC TEC can save up } \\
\text { to } 40 \text { euros per team in a year." (for profile 6) }\end{array}$ \\
\hline
\end{tabular}

In Figure 20, an overview of the behavior predictor is presented. In terms of time, there are three execution phases: day-ahead, when predictions are made and opportunities identified; real-time, when messages are sent; and day-after, when the impact of the messages is evaluated.

Each of the building blocks in Figure 20 is now briefly described.

Day-ahead stage: this code is run daily at midnight;

Load Forecast: values of energy for the model under assessment (either plugs, lights, or HVAC)—see Section 6.1 for more details;

Occupancy forecast: binary information concerning the occupancy of the room-see Section 6.3 for more details;

Temperature forecast: outdoors temperature forecast (forecasts retrieved from http: / / weather-service.enerapp.com, accessed on 15 February 2021);

Opportunities Forecast: this is the core of the behavior predictor, which identifies the opportunities for energy saving. It should be noted that the process for training the decision trees is fully automated. More info on this topic is given below;

Forecast impact of opportunities: takes the historical reaction to stimuli and calculates the predicted impact. This refers to savings in percentage and is calculated as a weighted average using the following rule: $50 \%$ for the average of all previous reactions to the previous $+50 \%$ of the reaction to the last message. More info on this topic is given below in the day-after stage;

Message selection: to avoid sending too many messages to the app users, this module takes the many opportunities identified by the behavior predictor to select only one of them to be sent. Typically, messages that have the highest potential for savings are selected; however, to avoid having the user receiving always the same kind of alert, the ones that are never or rarely selected are occacionally chosen to induce some variety; 
Real-time Stage: this code is run every hour and checks whether there is an alert for any room.

Outdoors temperature in real time: self-explanatory

Room occupancy: inferring data from the multisensor; this step performs verification of the actual room occupancy (see Section 3.3).

Send: the message is sent if the conditions presented in the Table 1 are met.

Gamification Platform: in this block, communication between the behavior predictor and the gamification platform is performed via a database using MySQL language.

Day-after stage: This code runs at the same time as the day-ahead stage.

Assess the impact of the previous-day message: this impact is returned in percentage, taking it as a ratio between the verified load and forecasted load. If the actual load is higher than the forecasted load, the values are disregarded because that would mean negative savings.

The behavior predictor communicates with the gamification platform the impact of the message so that the behavior is transformed into bonuses for the user (see Section 5).

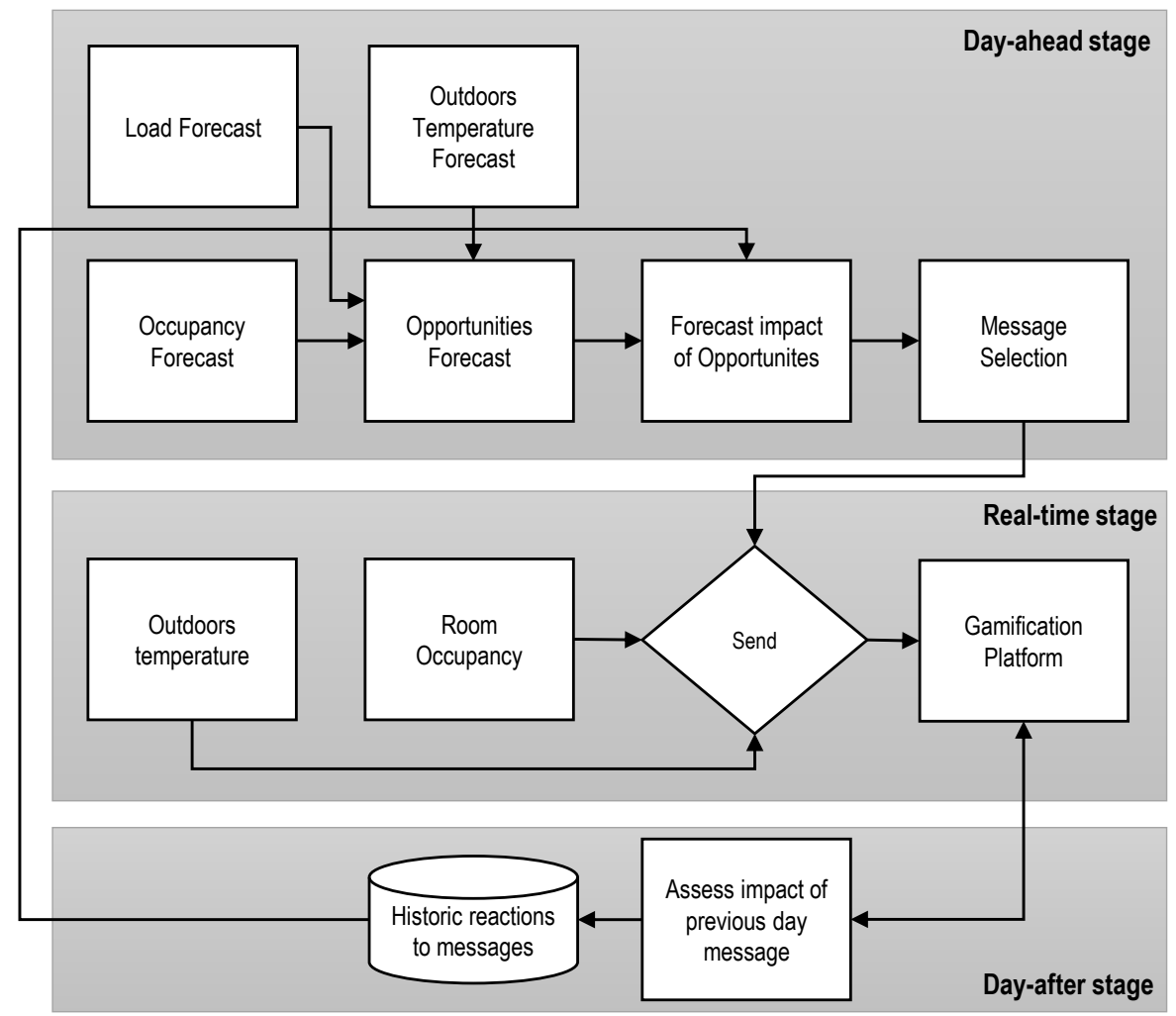

Figure 20. Behavior predictor module.

Here, further details regarding the machine learning models are provided. It should be noted that they can be trained online, i.e., the models are trained as many times as intended with the most recent data, in opposition to offline training in which the models are only trained once. The chosen machine learning methodology was decision trees because this is a binary classification problem and decision tree outputs are easy to interpret (a phenomenon known as white box). Decision tree classifiers have been applied for diverse domains such as intrusion detection, energy modelling for buildings, generating catalogs in e-commerce, image processing, diagnosis in medicine, fault detection in quality control, detecting lane boundaries in intelligent vehicles, and pattern recognition in remote sensing [43]. The implementation was done in Python using scikit-learn [25]. The methodology followed is found in [44]. Essentially, to create these models, it is necessary to access stored metered data (see Section 3.2) and to rely on multisensor (Section 3.3) data to obtain the load and occupancy ground truth. A second step is to detect outliers in these measurements and to 
remove them from training. The third step is to define what constitutes an opportunity, and here, we relied on statistical measures (e.g., we assume thresholds based on quartiles of historic consumption data). The training is done using a train-test split of $70 \%-30 \%$, using cross-validation of 10, and the parameters of the tree classifier are estimated resorting to grid search. Scoring is done using "recall" as a metric because the goal is to identify as many true positives as possible.

Implementation of the code is done using Python 3. The computation time for each team in the competition is in the order of $20 \mathrm{~s}$ for the day-ahead code and $0.02 \mathrm{~s}$ for the real-time code, when running on a Intel x64 Core i7-2600 CPU @ $3.40 \mathrm{GHz}$ with 8 GB of RAM.

\subsection{Automation Manager}

The automation manager application aims to produce optimized schedules to operate controllable loads. Heating, ventilation, and air conditioning (HVAC) is chosen as the target load to employ optimization techniques. The underlying reason for this choice is that HVAC is the largest energy consumer in both residential and tertiary buildings [45]. Therefore, applying intelligent control to manipulate the HVAC load can provide substantial potential energy savings. For more details, on the state-of-the-art and various HVAC-control methods existing in the literature, an interested reader is referred to the review works of $[46,47]$. The goal of the current application is to produce the most energy-efficient day-ahead schedules without compromising the users' comfort. The optimization process focuses on generating optimal timetables for heating and cooling systems, respectively. Once computed, these timetables are supplied to building managers and household owners for verification and deployment. If the building has direct automated control of loads, the manager can apply schedules to the equipment through computer interfaces. Otherwise, the produced optimal schedules become operational guidelines for dedicated personnel to manually switch on and off the systems at the required time.

Figure 21 represents the input and output data streams of the automation manager algorithm, which is detailed in [41]. There are four main types of incoming information required to produce HVAC schedules. First, the building metadata need to be collected. It includes the building's surface, preferred cooling and heating set-points, and heat transfer coefficients. The latter are often absent from the building's envelope. Therefore, additional methods to infer those coefficients responsible for thermal losses and thermal gains must be applied. Second, the weather data are recorded, namely the outdoor temperature and solar radiation. As those parameters are collected hourly, this defines the time resolution of the algorithm. Third, occupancy information is considered for two reasons. On the one hand, it allows us to estimate heat gains from people and to calculate the building's thermal load in a more precise manner. On the other hand, knowing when occupants are present in a specific space provides an opportunity to provide heating or cooling only where it is necessary. Avoiding using HVAC in unoccupied areas becomes the main contribution to potential energy savings. Fourth, the algorithm takes the HVAC demand of the building into account. It helps to provide the baseline for building's operation and can be useful to infer thermal coefficients if those are absent.

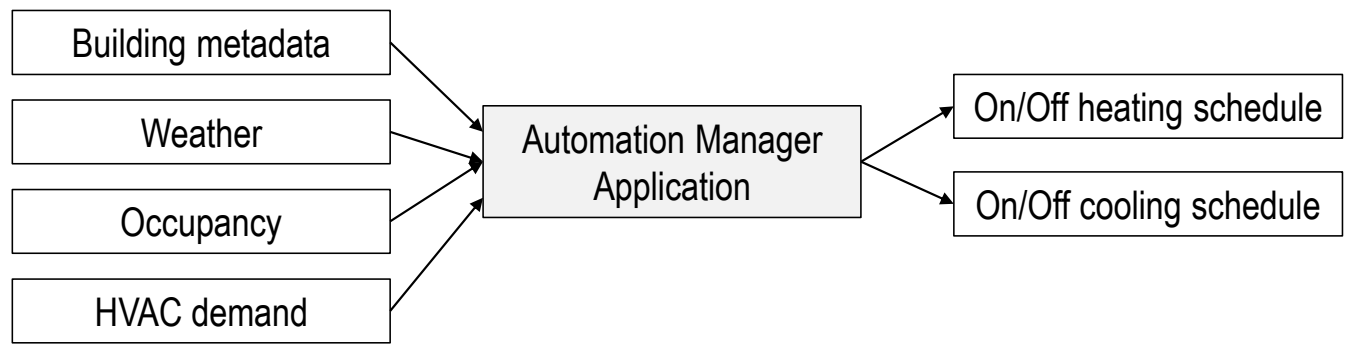

Figure 21. Block scheme of the automation manager application. 
The algorithm at the core of the automation manager application functions according to the set of predefined rules. These rules can be described as the following:

- If the building's thermal load is positive, the heating should be turned on. If it is negative, cooling is on.

- On and off switching is organized according to the set-point temperatures. If the outdoor temperature is lower or equal to the heating set-point, the heating is on. If it is higher or equal to the cooling set-point, cooling is on.

- If there is at least one occupant present in a space, heating or cooling might be turned on. If the space is empty, both systems are turned off to avoid energy waste.

- No simultaneous operation of heating and cooling systems is allowed. This rule means that the space cannot be cooled down and heated at the same time, as it does not make sense from a thermodynamics point of view.

- Outdoor temperature evolution should be taken into account. If its fluctuations are short-term and are not significant in their order of magnitude, no intermediary on/off switch should be realized. Instead, the guidelines should be kept similar to the ones in the previous time window. This rule helps prevent irrelevant alternations between heating and cooling systems and their states. Additionally, it allows us to extend the lifetime of the equipment and to avoid unnecessary maintenance.

The proposed HVAC automation algorithm demonstrated $15.4 \%$ of potential energy savings on the test dataset collected from the Porto demonstration site [41]. Further extensions to the algorithm should consider the HVAC system ramp-up times by introducing preheating and precooling periods. The length of such periods can be determined dynamically by taking into account the initial indoor temperature conditions and anticipated occupancy at the workspace. Realizing such an extension reduces the thermal discomfort of occupants to a minimum. Another option for improvement is to increase the time resolution of the algorithm. It allows us to consider more frequent changes in the occupancy status and environmental conditions of space. Therefore, the energy management strategy for controllable loads can become even more efficient and can potentially produce higher energy savings.

\subsection{Energy Manager}

The energy manager application is a display used for the Facility Managers (FM) and the Game Master (GM) as a support decision tool. The app objective is to display short messages generated in the FEEdBACk back-end modules that help the FMs and GMs understand what happens in the facility and to the teams.

These short messages can be related to long- or short-term actions depending on the action urgency or implementation complexity. Short-term scope messages (from now on, notifications) are generated in the gamification platform, while long-term scope (from now on, recommendations) are generated in the recommendations engine.

The information required from both the gamification platform and energy manager engine to generate the messages comes from the monitoring platform database, which is filled with FEEdBACk project apps and metering devices data. The interaction complexity is quite high in terms of data sharing. However, in the simplified chart presented in Figure 22, the interconnection can be observed. 


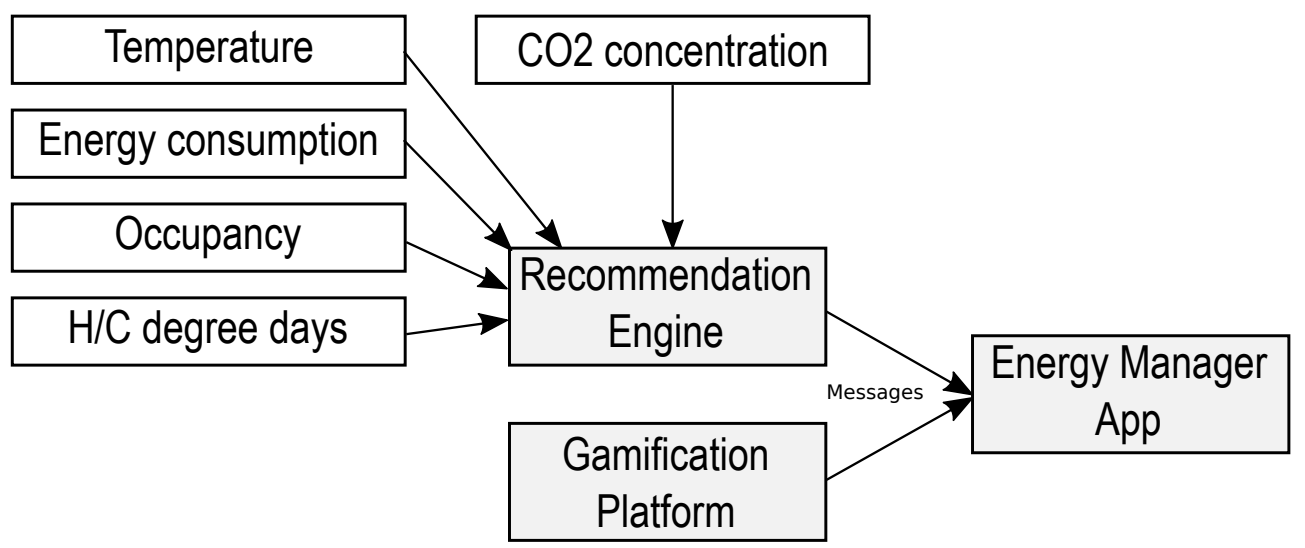

Figure 22. Block scheme of the energy management app.

The energy manager app solution was designed to cover two real needs observed during the project definition: (a) the energy savings not related to human behavior and (b) the energy savings not achievable due to the incapacity to perform an action by the end-users.

To cover the first need, we give the FM suggestions on how energy can be saved according to changes in the facility. The idea was to run historic information into algorithms to decide which changes are more suitable for solving recurrent issues. The problems could also be related to air quality. The expected outputs are messages that match the facility's needs and come from a backlog list that include valuable information, such as expected savings ratios.

The second problem is related to the end-users capability to perform energy-saving actions; it often happens that some actions related to energy consumption cannot be carried out by the end-users. For instance, HVAC settings in most Barcelona pilot buildings are only controllable by the FM. As the mobile app user targets are common employees, the energy manager app is used as a display to inform the FM when actions are not assigned to any of the end-user groups.

\section{Conclusions and Future Work}

The FEEdBACk project proposed a framework that promotes, stimulates, and delivers energy efficiency through behavioral change. To encourage more efficient energy utilization and more responsible consumer behavior, a gamification platform was developed to motivate behavioral change by fostering awareness and consumer engagement through a pervasive application that analyses context, sends personalized messages, and manages gamified peer competition and feedback.

The FEEdBACk solution was planned for implementation at three demonstration sites, in Porto (Portugal), Barcelona (Spain), and Lippe (Germany). A competition was envisioned for exploiting the gamification platform in these sites, but due to the COVID-19 pandemic, office buildings were left empty and the competition was never fully implemented. Therefore, the platform was not fully implemented and, for this reason, there are no results available yet to validate the value proposition of the project. Even so, several simulations were run using pre-COVID-19 historical data to validate the developed applications and hardware, which prove the value of the work developed.

The project yielded a diverse set of relevant outputs:

- A set of state-of-the-art algorithms fully integrated and prepared to run in real-time that allows for forecasting energy consumption, PV production, buildings occupancy, energy baselines, and building users' behavior.

- A low-cost multisensor for temperature, humidity, luminosity, and $\mathrm{CO}_{2}$ concentration levels that revealed a good performance working in a standalone mode (wireless and with a built-in battery) and conveying measurements in real-time to a database from the project. 
- Innovative social sciences techniques integrated into computational applications for users segmentation, which resulted in 6 distinct user profiles that were extensively described, allowing for better adjustment of the engagement and gamification activities to the interests of the citizens.

- An eclectic ICT platform capable of collecting, treating, storing, and displaying to the user all the relevant data with the overarching goal of increasing energy efficiency. The high flexibility of the ICT platform enables its straightforward implementation in a variety of contexts, whether they are different types of buildings (residential, office, or public buildings), different countries (a multi-language feature was implemented), or even buildings with different sensing levels (only one sensor and one meter are required per team).

- A mobile application for iOS and Android that serves as a front-end for the ICT platform and that enables interaction with the users. It incorporates a diversified set of multimedia content, games, quizzes, and informative charts in a peer competition environment, with the aim of raising engagement and awareness and of promoting behavioral change that is sustained with time.

The high flexibility of the ICT platform and all the attached components facilitates the scalability and replicability of the project outputs and their exploitation in the future by different stakeholders, such as the following:

- $\quad$ Building owners, who can use the platform to minimize energy cost by increasing energy efficiency and by reducing overall consumption.

- $\quad$ Energy services companies, who may install meters and sensors and use the platform also to increase energy efficiency and to reduce overall consumption.

- $\quad$ Aggregators/Retailers, who can use the platform to adjust energy consumption to market prices.

- Electricity network operators, who can use the platform to change electricity consumption profiles and adjust it to the electricity grid needs.

As future work, the consortium intends to exploit the full FEEdBACk platform in a real-world environment to collect enough data to validate both the individual applications and the fully integrated solution. Additionally, a set of papers are being prepared, which will exploit the results obtained in the most promising and innovative apps developed in the project, such as occupancy forecasting and the behavior predictor.

Author Contributions: Conceptualization, A.M., A.C., F.S., M.D. and N.W.; methodology, A.P., F.C., F.R., J.V., N.W., J.A. and T.S.; software, A.P., A.C., F.C., F.R., J.V., N.M. and J.A.; validation, A.M., A.C., F.S., M.D. and N.W.; formal analysis, F.C., F.R., J.V., N.M., J.A. and T.S.; investigation, F.C., F.R., J.V., N.M., J.A. and T.S.; resources, A.P., A.C. and T.S.; data curation, A.P. and A.C.; writing-original draft preparation, A.P., A.B., F.C., F.R., J.V., N.M., J.A. and T.S.; writing-review and editing, F.S., M.D. and N.W.; visualization, A.B., F.C., F.R., J.V., M.D., N.M. and J.A.; supervision, A.M., A.C., F.S. and N.W.; project administration, A.M., F.S. and N.W.; funding acquisition, A.M., F.S. and N.W. All authors have read and agreed to the published version of the manuscript.

Funding: This project received funding from the European Research Council (ERC) under the European Union's Horizon 2020 research and innovation programme (grant agreement $n^{\circ} 768935$ ).

Institutional Review Board Statement: Not applicable.

Informed Consent Statement: Not applicable.

Data Availability Statement: Data available on request due to restrictions. The data presented in this study are available on request from the corresponding author. The data are not publicly available due to use of personal data and the fact that project is still ongoing.

Conflicts of Interest: The authors declare no conflict of interest.

\section{Appendix A. Project Partners}

The project partners the following: 
- INESC TEC - Instituto de Engenharia de Sistemas e Computadores, Tecnologia e Ciência (Portugal): private non-profit research institution, dedicated to scientific research and technological development, technology transfer, advanced consulting and training, and pre-incubation of new technology-based companies.

- LiMETOOLS-Library of Interactive Media for Enterprise (United Kingdom): established in 2013 to develop interactive media-based online-training tools that would trigger behavior change in favor of more sustainable living and lower carbon-based business and policy.

- DEXMA Sensors (Spain): serving over 1500 businesses in more than 40 countries; DEXMA is a leading provider of Energy Management Software solutions for the commercial and industrial sector.

- ERF-Estudi Ramon Folchi I Associats SL (Spain): independent consulting company on energy, environment, and sustainability with 23 years of experience at an international and national level.

- $\quad$ IN-JET-In-JeT Aps (Denmark): small, medium-size enterprise established in 1997 and developed to become a renowned research and innovation firm in the field of technology development within internet-based and energy-related services.

- KREI KIPPE—Kreis Lippe Der Landrat (Germany): Municipal Administration founded in 1973. Currently, it is one of the biggest employers in the Lippe region, with around 5000 employees and apprentices in different fields, with an annual income of $700 \mathrm{mil}-$ lion Euros.

- TUDelft-Delft University of Technology (The Netherlands): the oldest, largest, and most comprehensive technical university in the Netherlands.

- EPFL-Ecole Polytechnique Fédérale de Lausanne (Switzerland): one of the two Swiss Federal Institutes of Technology. With the status of a national school since 1969 , the young engineering school has grown in many dimensions, to the extent of becoming one of the leading European institutions of science and technology.

\section{Appendix B. Demonstration}

The demonstration sites participating in the FEEdBACk project are from three different European regions:

- $\quad$ Porto (Portugal),

- Barcelona (Spain),

- $\quad$ Lippe (Germany).

\section{Porto}

The Porto test site for the FEEdBACk project is the headquarters building of INESC TEC, as shown in Figure A1.

It is located in the campus of the Faculty of Engineering of the University of Porto (FEUP) in the northern boundary zone of the city. This location is home to one of the three university centres of Porto and has in its vicinity several roads and train stations that allow for a quick connection to the city centre as well as the northern part of the country.

The INESC TEC headquarters consists of two contiguous building sections built in different years (the first one dates from 2002 and the most recent one dates to 2012) but with similar structure, internally and externally. The buildings consist of six floors, where one of them is below the ground floor. The combined building has a total area (usable) of about $4000 \mathrm{~m}^{2}$ with a daily occupation of about 400 users. Although the facilities are open $24 / 7$, most of the users have work between 9 a.m. to 7 p.m. on weekdays. 


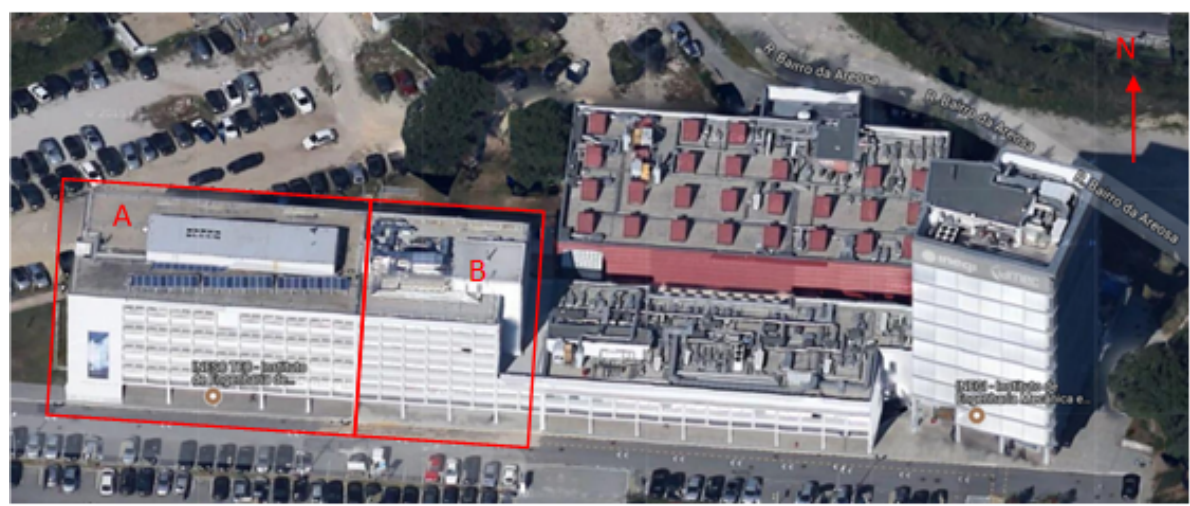

Figure A1. INESC TEC buildings.

\section{Barcelona}

The Barcelona demonstration site is located in the town of "El Prat de Llobregat", about 10-15 km southwest of the city of Barcelona (see Figure A2). The "Llobregat" river is the northeastern boundary of the town and the C-31 highway, leading to the airport, which is the northwestern boundary of the town. The greater municipality of "El Prat del Llobregat" incorporates agricultural areas, parts of the delta of the Llobregat river, and the beach-front with the Mediterranean.

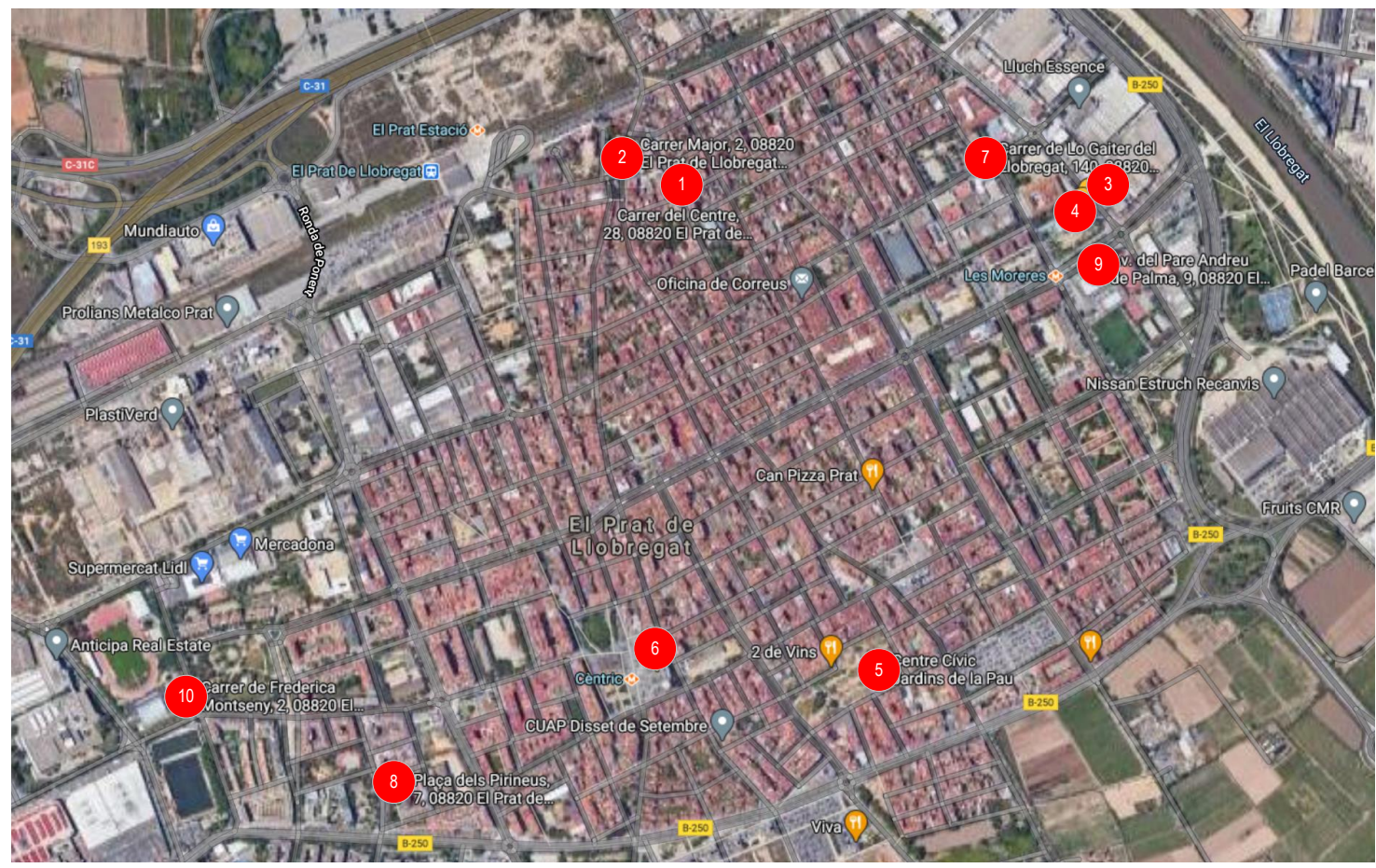

Figure A2. An aerial view of "El Prat del Llobergat" highlighting the locations of the 10 different buildings of the Barcelona demonstration site.

The population of the municipality is about 64,000 inhabitants, and it is concentrated in the town. Ten buildings owned by the Municipality of Llobregat ("Ajuntament del Prat Llobregat") were chosen from the demonstration site. 
Table A1. List of buildings of Barcelona test site.

\begin{tabular}{llll}
\hline Number & Name of Building & Type of Building & Building Floor Area \\
\hline 1 & Municipal Offices, carrer Centre 26-30 & Office & $2067 \mathrm{~m}^{2}$ \\
2 & Municipal Offices, carrer Major 2-4 & Office & $1630 \mathrm{~m}^{2}$ \\
3 & Economic Promotion Centre & Office & $2238 \mathrm{~m}^{2}$ \\
4 & Cultural centre "La Capsa" & Cultural centre & $1500 \mathrm{~m}^{2}$ \\
5 & Community centre "Jardins de la Pau" & Cultural centre & $1176 \mathrm{~m}^{2}$ \\
6 & Cultural centre Centric & Cultural centre & $14,972 \mathrm{~m}^{2}$ \\
7 & "Delta del Llobregat" Job Training School & Education centre & $2238 \mathrm{~m}^{2}$ \\
8 & Adult school "Terra Baixa" & Education centre & $860 \mathrm{~m}^{2}$ \\
9 & CEM "Estruch" & Sport centre & $8528 \mathrm{~m}^{2}$ \\
10 & CEM "Sagnier" & Sport centre & $5700 \mathrm{~m}^{2}$ \\
\hline
\end{tabular}

\section{Lippe}

The village Dörentrup (Figure A3) in the district of Lippe is one of the demonstration sites for the FEEdBACk project.

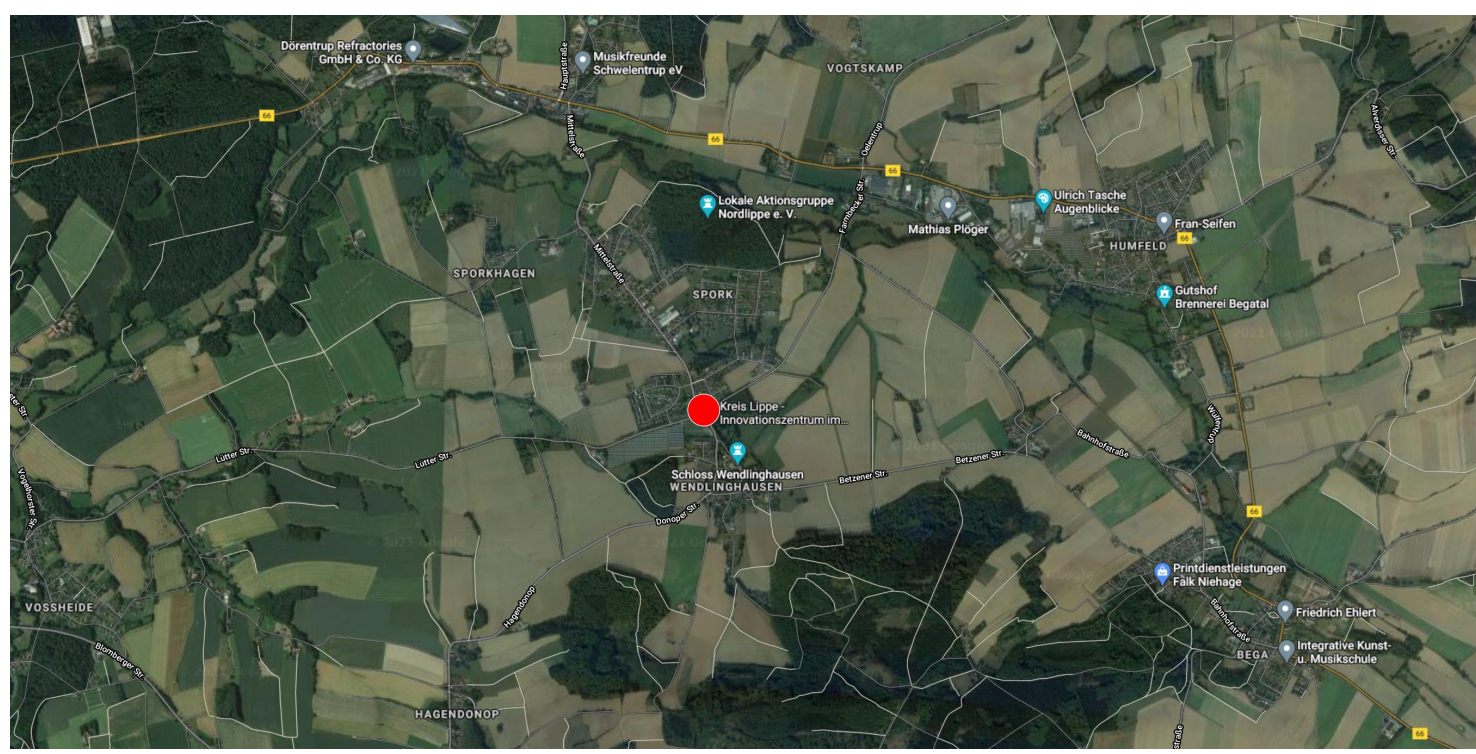

Figure A3. Lippe demo.

The district of Lippe is located in the northeast of North Rhine-Westphalia. About 7900 inhabitants live in Dörentrup, and the demo site consists of about 30-40 residential buildings in the village.

In the municipality of Lippe, around a quarter of the electricity consumption is private households. In this sector, the increasing energy efficiency of devices and the changing user behavior has experienced changes in recent years.

\section{Appendix C. FEEdBACk Competition}

In order to implement the gamification platform (Section 5), two complementary competitions were envisioned: one individual competition and one collective competition. The individual ranking reflects the user engagement: amount of quizzes and respective results, amount of watched videos, won badges, among other indicators retrieved from the mobile app (Section 5). On the other hand, the collective competition reflects the amount of conserved energy without compromising the user comfort level. In order to monitor the comfort associated with each team, one sensor was assigned to each of them.

The building users were assigned to teams according to the area they use. For example, in INESC TEC, 14 teams were created: one team per open space, one per laboratory, and 
one per row of offices (as detailed in Section 3.2). In Barcelona, the same logic was applied, whereas in Lippe, each house is a team.

The advantages of creating a competition with teams are as follows:

- Due to the implementation, General Data Protection Regulation (GDPR) concerns could be raised due to tracking consumption of an individual;

- The resolution of metering is limited to a whole open-space or offices (see Section 3.2), so it would not be feasible to track most components of energy consumption of a single individual;

- Inducing competition between teams can be seen as a driver for increasing engagement through a sense of team building and collective belonging of individuals that might otherwise not be so inclined to use the app.

In order to engage building users in the gamification app, a list of prizes was envisioned. In order for the prizes to be meaningful in the context of the project, their value would need to be related to the amount of achieved energy conservation. Therefore, having in mind that the FEEdBACk solution allows for measuring the impact of the behavior change (see Sections 2.1 and 6.1), the first step would be to monetize the conserved energy by comparing real energy consumption with the forecasted baseline. The significant amount of $50 \%$ of the monetized savings provided by FEEdBACk solution would be subject to a participatory budget, in which the users would be able to vote for measures to be implemented after the end of the project.

At individual level, there would be prizes such as free meals in the INESC cafeteria, vouchers, and other relatively small value prizes that would be provided on a weekly basis for the best player that week. These prizes would represent $25 \%$ of the remaining monetized saving. In order to sustain engagement in the long run, achievements such as concluding a level or obtaining a rare badge would be devoted to the remaining $25 \%$ and would be awarded prizes in this category including an extra half day holiday, the possibility of using the laboratory electric vehicle (Renault Twizzy) for one weekend, obtaining a premium parking place for a limited time, and finally having a dedicated article in the INESC TEC magazine.

The reasoning of prizes in Barcelona was similar in spirit.

Regarding Lippe, the attribution of prizes was up to the municipality and the competition would be between households. Every three weeks, the top three ranks would receive prizes.

As stated in Section 2.2, the competition was implemented in Porto but had to be halted due to COVOD-19, whereas in Barcelona and Lippe, it was not fully implemented.

\section{Appendix D. Profile Segmentation Questionnaire}

For the questions related to energy and pro-environmental behavior, a 5-point Likert scale was applied using the strongly disagree to strongly agree continuum with a mix of positive and negatively worded statements. 
Table A2. Profile segmentation questionnaire.

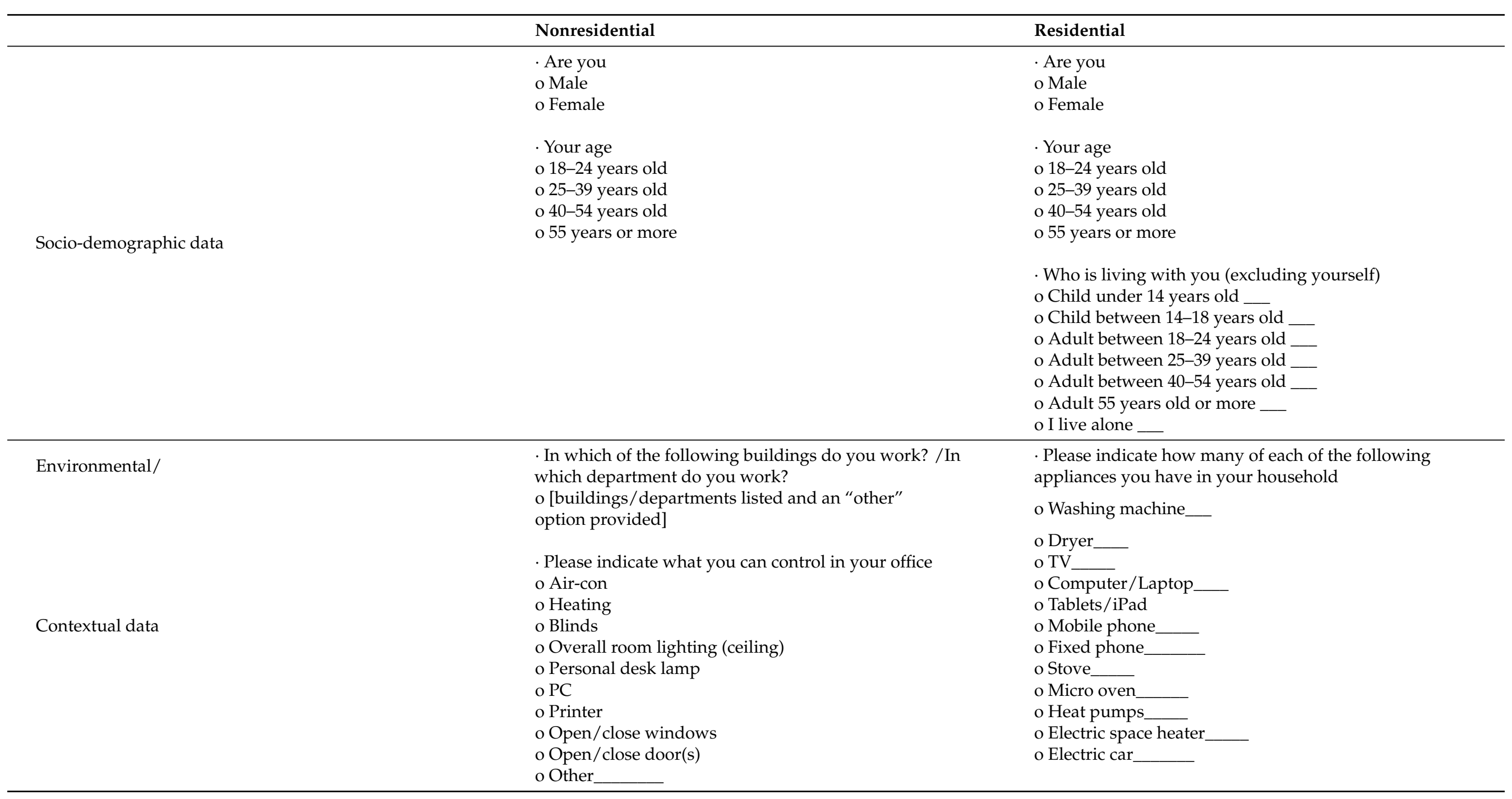


Table A2. Cont.

Please indicate the main location for your workstation

o Own private office

o Shared closed office

o Open office

o Other

How many people do you share office/room with?

o Less than 2

o $2-4$

o $5-8$

o More than 12

Behavioral Beliefs-Attitude Toward the Behavior

o Strongly agree

o Agree

o Neither agree nor disagree

o Disagree

o Strongly disagree

Energy conservation means I have to work/live less comfortably.

Energy conservation will restrict my freedom.

Protecting the environment is a very important issue.

- Reducing my energy consumption at work/home will not have a significant positive effect on the environment.

I am very concerned with the overall negative effects of human behaviors and consumption on the environment.

The state of my local environment is important for my own well-being and health

- I think I should save more energy at work/home.

Normative Beliefs - Subjective Norm

- My colleagues/family or friends think that I should save more energy at work/home

- Most people I know take actions in helping to protect the environment.

o Strongly agree

o Agree

- Generally, there is a feeling at/in my work place/neighbourhood to promote environmentally protective behaviors.

o Neither

- It is not worth me doing things to help the environment if others don't do the same

o Disagree

o Strongly disagree

Control Beliefs - Perceived Behavioral Control

I am interested in my energy consumption at work/home

o Strongly agree

o Agree

I can reduce my energy use quite easily.

I know how I can save energy.

I think there is little I can realistically do to reduce how much energy I use at work.

o Neither agree nor disagree

o Disagree

I'm very well informed about the possibilities to protect the environment.

- I'm interested in possibilities of saving energy at work/home

o Strongly disagree

I'm prepared to save energy with the right support 


\section{Appendix E. Profile Segmentation In Detail}

\section{Appendix E.1. Profile 1 Description}

This group shows high environmental awareness, and they are concerned about the environment and about the negative effects on the environment, both locally and globally, caused by energy consumption. They have a very good awareness and understanding of the general causes and effects as well as what to do to help minimize the negative effects of human consumption on the environment. They see a clear link between the state of the environment and human health, and their concern for the environment is re-enforced by a concern for the negative effect of pollution on personal health and vice versa. They act as pro-environmental as possible because it is regarded as having a direct causal benefit to their own health.

They have a high inclination (intention) and motivation to act pro-environmentally and to save as much energy as they can, and they will do so because they find it manageable and because they personally want to contribute-even if they think they do more than others. They are aware that they probably belong to a small group with pro-environmental behaviors, and the social norms they associate themselves with are very strong. They think that they are very well informed about environmental issues, including causes and effects as well as the solutions, and therefore it is hard, if not impossible, for them not to incorporate some pro-environmental habits into their lifestyle. Certainly, reducing their energy consumption is considered a very manageable behavior. Being pro-environmental is an important part of their personal and social identity, and it makes them feel good. They also feel that pro-environmental behavior will always have a positive effect on their lives, their local community, and globally. They understand how to be pro-environmental and have faith in that they know what to do, that they are able to do it, and that they are able to make a difference. They are motivated by reinforcing their sense of being altruistic and taking a lead position in the quest to protect the environment (being a role model) to the extent of seeing themselves as better and more successful (in saving energy) than most people.

Main characteristics:

- Aware and knowledgeable about environmental issues

- Great concern about environmental issues as they see a direct link between the health of the environment and their personal health

- Feeling good about themselves for doing their bit (saving energy)

- Confident about their ability to reduce their energy consumption

Motivational drivers:

- Acknowledge their contribution and positive effect on the local—and global—environment and show concrete factual awareness of this. Focus on the positive.

- Show that they can inspire others to follow in their footstep, particularly that every little thing helps and matters-and that someone has to lead.

- Boost their feeling of altruism.

Motivational barriers:

- Negative facts on state of the environment; focusing on the negative rather than on the positive actions they do and how successful they are

- Practical and external barriers that affect their success, particularly frustration as they know what to do, believe that they can do it, but external barriers (e.g., cannot control the installations) stop them from being who they feel they are (i.e., pro-environmental and conscientious and sustainable consumers).

\section{Appendix E.2. Profile 2 Description}

This group show a high environmental awareness, and they are concerned about the environment and about the negative effect on the environment, both locally and globally, caused by energy consumption. They have a very good awareness and understanding of the general causes and effects as well as what to do to help minimize the negative effects of 
human consumption on the environment. They see a clear link between the state of the environment and human health, and their concern for the environment is re-enforced by a concern for the negative effect of pollution on personal health and vice versa. They act as pro-environmental as possible because it is regarded as having a direct causal benefit to their own health.

It is their doubts about what is actually possible and what can they actually do that makes them significantly different from Profile 1 . They have a somewhat high inclination (intention) to act pro-environmentally and to save as much energy as they can, but they still think that it is not that simple to do. They are aware that they probably belong to a small group with pro-environmental behaviorsm and the social norms they associate themselves with are very strong. They do not care so much about what other people do, or do not do, and this does not affect their intentions or motivations to any significant degree. They will do what they find manageable and what they think they can succeed in doing. They are less secure in their ability to implement pro-environmental behaviors and/or to change their current energy consumption behaviors. This lack of belief in themselves and how much control and affect they have in reality, both on a small and a large scale, is mainly linked to some doubts about the concrete actions or behavioral changes they need to and are able to make. It could frustrate them that, while they are very concerned about the environment and see themselves as pro-environmental, not least because they feel it is the social norm, they are not confident that they can successfully change their behaviors and actually make a difference even if they realize that it would not come with great personal sacrifices in terms of comfort or their current lifestyle.

They are likely to feel that their success in reducing their energy consumption is determined by the level and type of support they can get and the external structures (easy opportunities to reduce energy) are in place. They may also have some concern that the external structure actually works as a barrier to their efforts to reduce energy and act more pro-environmental in general, e.g., that the building's overall energy system and structure is poor (such as being poorly isolated or have old installations that consume a lot of energy) and/or is poorly managed.

Main characteristics:

- Aware and knowledgeable about environmental issues

- Great concern about environmental issues as they see a direct link between the health of the environment and their personal health

- Insecure about what they can actually do and if they can succeed in making changes. Motivational drivers:

- Acknowledge their contribution and positive effect on the local—and global—environment and show concrete facts hereof. Focus on the positive.

- Concrete examples/suggestion of small manageable behavioral changes (one step at a time approach) and use success and concrete results to show them that they can do it

- Increase their awareness of others who act pro-environmentally, and their results, so they are inspired by other success stories

- Create a strong feeling of community where everyone cheers on and support each other.

Motivational barriers:

- Negative facts on state of the environment; focusing on the negative rather than on the positive actions they do and how successful they are

- Overload of the many things they could possibly do; too many suggestions can be too overwhelming and may lead to a behavioral paralysis (inability to act)

- Lack of control of the appliances that consume energy

- Some distrust that efforts may be undermined by external structures

- $\quad$ Feeling alone and left to fend for themselves. 


\section{Appendix E.3. Profile 3 Description}

This group has a moderate pro-environmental attitude; they belong to the average. There are some issues (questions) that they are more favorable to than others but, overall, they tend to be quite neutral, leaning at times towards the more pro-environmental attitude and inclination. The pro-environmental attitude is strongest in relation to the perceived negative consequences for their own health and local community. They focus on the issues very much from a very personal perspective and in a personal context.

They tend to act based on personal principles and feelings and are therefore not affected by what others do (or do not do); they believe that they can make a difference, can contribute to a more sustainable energy consumption, and can help protect the environment, and this belief is a driving force. Their belief in themselves as being capable of reducing their energy consumption is stronger than their environmental concern and their intention to behave more pro-environmentally. It is therefore not a question of them not being able to change their behavior but more of choosing not to do so, either because their concern for the environment is not great enough and/or because they are not willing to make personal sacrifices and/or they are not confident that their own personal behavior has a great impact (negatively or positively) on the environment. They therefore have a more narrow and personalized view on the environment in the sense that they focus on their own life, actions, and habits. In other words, they also do not see themselves as contributing to the problem because they feel they do what they can and they do it well. They are likely to characterize themselves as realistic about what they actually can do, even if they see themselves as doing more than most other people or doing what they can despite other people not doing the same.

Main characteristics:

- A moderate awareness and knowledge about environmental issues

- A moderate concern about environmental issues

- They are very confident in their abilities to change their own behavior and to reduce energy.

Motivational drivers:

- $\quad$ Focus on the limited restraints changing behaviors will have on their daily lives (comfort, freedom); let them experience it rather than tell them

- Confirm that their efforts do matter (concrete facts) on a more global scale

- Appeal to their sense of being a good person (altruism)

- Focus on their abilities and capabilities to implement behavioral changes, one small change at a time, and celebrate success

- Strengthen their sense of being in/acting together in a group (peer pressure and group support) and being part of a global community.

Motivational barriers:

- Cannot see the correlation between the local and the global

- Too noticeable impact on their daily lives (comfort, freedom)

- Unless they win, competing with others could be de-motivating as it could alter their strong sense of self-efficacy.

\section{Appendix E.4. Profile 4 Description}

This group has a moderate pro-environmental attitude; they belong to the average. There are some issues (questions) that they are more favorable to than others but, overall, they tend to be quite neutral, leaning at times towards the more pro-environmental attitude and inclination. The pro-environmental attitude is strongest in relation to the perceived negative consequences for their own health and local community. They focus on the issues very much from a very personal perspective and in a personal context.

However, they are not that confident either about their opportunities, necessary knowhow, or their capabilities to reduce their energy consumption nor the effect it will have for them personally or in the grander scheme of things (work place or globally). They are less 
secure in their ability to implement pro-environmental behaviors and/or to change their current energy consumption behaviors. This lack of belief in themselves and how much control and affect they have in reality, both on a small and a large scale, is mainly linked to some doubts about the concrete actions or behavioral changes they need to and are able to make. They would like more support, knowledge, and concrete opportunities (actions to do) if they are going to make any changes.

They may feel that there are too many things that are out of their control, so that, even if the intention to act is there, they focus on the real or perceived external barriers that affect what they can do and achieve. Some may even feel quite helpless or powerless. Some people will feel that they are far more realistic about what they can do to reduce energy precisely because they are acutely aware of the external barriers that hinder them in having full control of their behaviors.

Main characteristics:

- A moderate awareness and knowledge about environmental issues

- A moderate concern about environmental issues; strongest in relation to their own self/personal lives

- Not sure what to do, how to do it, and if they can do anything that matters.

Motivational drivers:

- Support; making energy reduction easy and simple and celebrate every little result

- Confirm that their efforts do matter (concrete facts) and focus on what they can control

- Strengthen their sense of being in/acting together in a supportive (not competitive) group

- Increase knowledge of what they can do and the reality of the perceived external barriers/constraints.

Motivational barriers:

- Competition with others could reinforce their weak sense of self-efficacy (unless they win)

- Lack of actual behavioral control

- Lack of information on energy consumption and celebration of their personal achievements (effects on consumption).

\section{Appendix E.5. Profile 5 Description}

This group's overall pro-environmental attitude and intentions for being more proenvironmental is below average; they are less concerned overall or they simply do not feel strongly about environmental issues. They do not feel that there is any incentive or culture in their work place/neighbourhood to act pro-environmental or to show interest and concern with these issues. It is simply not prioritized or an area of focus; in some cases, the opposite is the case. Lack of structures and facilities to be pro-environmental only reinforces this feeling. This group neither thinks it is worth doing anything if others do not do the same nor thinks that their individual actions or behaviors to reduce their energy consumption will have any significant impact, a belief which only reinforces a kind of laissez-faire attitude overall. They may find it hard to look at environmental issues in a global context, and as they do not feel affected in their daily lives, they cannot see that there are any grounds for concern and thus action.

Their sense of self-efficacy is generally moderate; it may also be stronger for some individuals, but this does not necessarily translate into changing behavior or acting proenvironmentally in general. They are likely to feel a sense of paralysis and be overwhelmed by the magnitude of the issues and the little impact their personal behaviors have in the grand scheme of things. Their dominant attitudes and perceptions are thus not affected to any significant degree on their feelings of self-efficacy.

Main characteristics:

- A moderate awareness and knowledge about environmental issues

- No immediate concern about environmental issues; somewhat indifferent. 
- Particularly influenced by a perception of what others do not do

- Too narrowly focused on themselves/their individual actions to see how "it" can add up.

Motivational drivers:

- Support; making energy reduction easy and simple and celebrate every little result

- Confirm that their efforts do matter (concrete facts)

- Increase their awareness of others who act pro-environmentally and promote the social norm

- Strengthen their sense of being in/acting together in a supportive group Motivational barriers:

- Acting alone or too isolated; seeing others not doing "their bit"

- High level information or preaching that is too distanced from their personal context

- Feelings of paralysis and inability to do anything worthwhile

- Focus on failures or shortcomings rather than success.

\section{Appendix E.6. Profile 6 Description}

This group's overall pro-environmental attitude and intentions for being more proenvironmental is below average; they are less concerned overall, or they simply do not feel strongly about environmental issues. They do not feel that there is any incentive or culture in their work place/neighbourhood to act pro-environmentally or to show interest and concern with these issues. It is simply not prioritized or an area of focus; in some cases, the opposite is the case. They may find it hard to look at environmental issues in a global context, and as they do not feel affected in their daily lives, they cannot see that there are any grounds for concern and thus action. This group neither thinks it is worth them doing anything if others do not do the same nor thinks that their individual actions or behaviors to reduce their energy consumption will have any significant impact, a belief which only reinforces a kind of laissez-faire attitude overall. If there is something they can do and which will make a difference, they would like to know. The people in this profile who are interested in knowing more about how to save energy are interested because they would like to increase their knowledge, not necessarily because they want to change their habits too much. They may still question how much it matters and if they can succeed. They tend to doubt that they can even obtain concrete tips or instructions or that these will have any significant impact. They are simply not confident that they can succeed or make a difference, and then again, why should they?

Main characteristics:

- Below awareness and knowledge about environmental issues

- No concern about environmental issues; somewhat indifferent and low inclination to be pro-environmental.

- Particularly influenced by a perception of what others do not do

- Too narrowly focused on themselves/their individual actions to see how "it" can add up (positively or negatively).

Motivational drivers:

- Inform and educate, in both a local and global context

- Support; making energy reduction easy and simple and celebrate every little result

- Show that their efforts do matter (concrete facts)

- Increase their awareness of others who act pro-environmentally and promote the social norm

- $\quad$ Strengthen their sense of being in/acting together in a supportive group

- $\quad$ Focus on external facilitators (e.g., reminders, access to control panels) and what they control individually.

Motivational barriers:

- External barriers limiting their control 
- Feelings of paralysis and inability to do anything worthwhile

- Lack of knowledge (local and global)

- Focus on failures or shortcomings rather than success.

\section{References}

1. Directorate-General for Energy-European Commission. Good Practice in Energy Efficiency: For a Sustainable, Safer and More Competitive Europe. 2017. Available online: https://ec.europa.eu/energy/sites/ener/files/publication/version2-web.pdf (accessed on 15 February 2021).

2. Yoshino, H.; Chen, S. Total Energy Use in Buildings: Analysis and Evaluation Methods (Annex 53). 2016. Available online: http://www.iea-ebc.org/Data/publications /EBC_PSR_Annex53.pdf (accessed on 1 October 2020).

3. Aslani, A.; Bakhtiar, A.; Akbarzadeh, M.H. Energy-efficiency technologies in the building envelope: Life cycle and adaptation assessment. J. Build. Eng. 2019, 21, 55-63. [CrossRef]

4. Wang, H.; Wang, S.; Tang, R. Development of grid-responsive buildings: Opportunities, challenges, capabilities and applications of HVAC systems in non-residential buildings in providing ancillary services by fast demand responses to smart grids. Appl. Energy 2019, 250, 697-712. [CrossRef]

5. Frederiks, E.; Stenner, K.; Hobman, E. Household energy use: Applying behavioural economics to understand consumer decision-making and behaviour. Renew. Sustain. Energy Rev. 2015, 41, 1385-1394. [CrossRef]

6. Simanaviciene, Z.; Volochovic, A.; Vilke, R.; Palekiene, O.; Simanavicius, A. Research Review of Energy Savings Changing People's Behavior: A Case of Foreign Country. Procedia Soc. Behav. Sci. 2015, 191, 1996-2001. [CrossRef]

7. Paone, A.; Jean-Philippe, B. The Impact of Building Occupant Behavior on Energy Efficiency and Methods to Influence It: A Review of the State of the Art. Energies 2018, 11, 953. [CrossRef]

8. Zehir, M.; Ortac, K.; Gul, H.; Batman, A.; Aydin, Z.; Portela, J.; Soares, F.; Bagriyanik, M.; Kucuk, U.; Ozdemir, A. Development and Field Demonstration of a Gamified Residential Demand Management Platform Compatible with Smart Meters and Building Automation Systems. Energies 2019, 12, 913. [CrossRef]

9. Reeves, B.; Cummings, J.J.; Scarborough, J.K.; Yeykelis, L. Increasing Energy Efficiency with Entertainment Media: An Experimental and Field Test of the Influence of a Social Game on Performance of Energy Behaviors. Environ. Behav. 2015, 47, 102-115. [CrossRef]

10. Deterding, S.; Dixon, D.; Nacke, L.; O’Hara, K.; Sicart, M. Gamification: Using Game Design Elements in Non-Gaming Contexts. In Proceedings of the 2011 Annual Conference Extended Abstracts on Human Factors in Computing Systems (CHI EA'11); 2011; pp. 2425-2428. Available online: http://gamification-research.org/chi2011/ (accessed on 15 February 2021).

11. Ajzen, I. Theory of Planned Behaviour. 2006. Available online: https://people.umass.edu/aizen/tpb.html (accessed on 18 December 2019).

12. Anderson, M. New Ecological Paradigm (NEP) Scale. Berks. Encycl. Sustain. 2012, 6, 260-262.

13. Ramkissoon, H.; Weiler, B.S. Place attachment and pro-environmental behaviour in national parks: The development of a conceptual framework. J. Sustain. Tour. 2012, 20, 257-276. [CrossRef]

14. Cordano, M.; Frieze, I.H. Pollution reduction preferences of US environmental managers: Applying Ajzen's theory of planned behavior. Acad. Manag. J. 2000, 43, 627-641.

15. Davis, G.; O'callaghan, F.; Knox, K. Sustainable attitudes and behaviours amongst a sample of non-academic staff. Int. J. Sustain. High. Educ. 2009. Available online: https://www.researchgate.net/publication/42622170_Sustainable_attitudes_ and_behaviours_amongst_a_sample_of_non-academic_staff_A_case_study_from_Information_Services_Department_Griffith_ University_Brisbane (accessed on 15 February 2021).

16. Greaves, M.; Zibarras, L.D.; Stride, C. Using the theory of planned behavior to explore environmental behavioral intentions in the workplace. J. Environ. Psychol. 2013, 34, 109-120. [CrossRef]

17. Armitage, C.J.; Conner, M. Efficacy of the theory of planned behaviour: A meta-analytic review. Br. J. Soc. Psychol. 2001, 40, 471-499. [CrossRef] [PubMed]

18. Kwasnicka, D.; Dombrowski, S.U.; White, M.; Sniehotta, F. Theoretical explanations for maintenance of behaviour change: A systematic review of behaviour theories. Health Psychol. Rev. 2016, 10, 277-296. [CrossRef] [PubMed]

19. Darnton, A. Reference Report: An Overview of Behaviour Change Models and Their Uses; GSR Behaviour Change Knowledge Review; Government Social Research Unit: London, UK, 2008. Available online: https://assets.publishing.service.gov.uk/ government/uploads/system/uploads/attachment_data/file/498065/Behaviour_change_reference_report_tcm6-9697.pdf (accessed on 15 February 2021).

20. Kullmuss, A.; Agyeman, J. Mind the Gap: Why do people act environmentally and what are the barriers to pro-environmental behaviour. Environ. Educ. Res. 2002, 8, 239-260. [CrossRef]

21. Sniehotta, F.F. Towards a theory of intentional behaviour change: Plans, planning, and self-regulation. Br. J. Health Psychol. 2009, 14, 261-273. [CrossRef]

22. Vaněk, J. New Ecological Paradigm as a Research Approach in the Czech Republic: An Analysis of Environmental Attitudes of Czechs. 2017. Available online: https://dspace.cuni.cz/handle/20.500.11956/86367 (accessed on 15 February 2021).

23. Ntanos, S.; Kyriakopoulos, G.; Skordoulis, M.; Chalikias, M.; Arabatzis, G. An application of the new environmental paradigm (NEP) scale in a Greek context. Energies 2019, 12, 239. [CrossRef] 
24. Scannell, L.; Gifford, R. The relations between natural and civic place attachment and pro-environmental behavior. J. Environ. Psychol. 2010, 30, 289-297. [CrossRef]

25. Pedregosa, F.; Varoquaux, G.; Gramfort, A.; Michel, V.; Thirion, B.; Grisel, O.; Blondel, M.; Prettenhofer, P.; Weiss, R.; Dubourg, V.; et al. Scikit-learn: Machine Learning in Python. J. Mach. Learn. Res. 2011, 12, 2825-2830.

26. Ke, G.; Meng, Q.; Finley, T.; Wang, T.; Chen, W.; Ma, W.; Ye, Q.; Liu, T. LightGBM: A Highly Efficient Gradient Boosting Decision Tree. In Proceedings of the Annual Conference on Neural Information Processing Systems, Long Beach, CA, USA, 4-9 December 2017.

27. Rothfuss, J.; Ferreira, F.; Walther, S.; Ulrich, M. Conditional Density Estimation with Neural Networks: Best Practices and Benchmarks. arXiv 2019, arXiv:1903.00954.

28. Andrade, J.R.; Bessa, R.J. Improving Renewable Energy Forecasting with a Grid of Numerical Weather Predictions. IEEE Trans. Sustain. Energy 2017, 8, 1571-1580. [CrossRef]

29. Hong, T.; Pinson, P.; Fan, S.; Zareipour, H.; Troccoli, A.; Hyndman, R.J. Probabilistic Energy Forecasting: Global Energy Forecasting Competition 2014 and Beyond. 2016. Available online: https:/ / orbit.dtu.dk/en/publications/probabilistic-energyforecasting-global-energy-forecasting-compet (accessed on 15 February 2021).

30. Nagy, G.I.; Barta, G.; Kazi, S.; Borbély, G.; Simon, G. GEFCom2014: Probabilistic solar and wind power forecasting using a generalized additive tree ensemble approach. Int. J. Forecast. 2016, 32, 1087-1093. [CrossRef]

31. Snoek, J.; Larochelle, H.; Adams, R. Practical Bayesian Optimization of Machine Learning Algorithms. arXiv 2012, arXiv:abs/1206.2944.

32. Nogueira, F. Bayesian Optimization: Open Source Constrained Global Optimization Tool for Python. 2014. Available online: https: // bibbase.org/network/publication/nogueira-bayesianoptimizationopensourceconstrainedglobaloptimizationtoolforpython2014 (accessed on 15 February 2021).

33. Hart, G.W. Non-intrusive appliance load monitoring. Proc. IEEE 1992, 80, 1870-1891. [CrossRef]

34. Faustine, A.; Mvungi, N.H.; Kaijage, S.; Michael, K. A Survey on Non-Intrusive Load Monitoring Methodies and Techniques for Energy Disaggregation Problem. arXiv 2017, arXiv:1703.00785.

35. Esa, N.F.; Abdullah, M.P.; Hassan, M.Y. A review disaggregation method in Non-intrusive Appliance Load Monitoring. Renew. Sustain. Energy Rev. 2016, 66, 163-173. [CrossRef]

36. Zoha, A.; Gluhak, A.; Imran, M.; Rajasegarar, S. Non-Intrusive Load Monitoring Approaches for Disaggregated Energy Sensing: A Survey. Sensors 2012, 12, 16838-16866. [CrossRef] [PubMed]

37. Holweger, J.; Dorokhova, M.; Bloch, L.; Ballif, C.; Wyrsch, N. Unsupervised algorithm for disaggregating low-sampling-rate electricity consumption of households. Sustain. Energy Grids Netw. 2019, 19, 1-23. [CrossRef]

38. Kleiminger, W.; Mattern, F.; Santini, S. Predicting household occupancy for smart heating control: A comparative performance analysis of state-of-the-art approaches. Energy Build. 2014, 85, 493-505. [CrossRef]

39. Kleiminger, W. Occupancy Sensing and Prediction for Automated Energy Savings. Ph.D. Thesis, ETH Zurich, Zürich, Switzerland, 2015.

40. Becker, V.; Kleiminger, W. Exploring zero-training algorithms for occupancy detection based on smart meter measurements. Comput. Sci. Res. Dev. 2018, 33, 25-36. [CrossRef]

41. Dorokhova, M.; Ballif, C.; Wyrsch, N. Rule-based scheduling of air conditioning using occupancy forecasting. Energy AI 2020, 2, 100022. [CrossRef]

42. Taylor, S.; Letham, B. Forecasting at scale. PeerJ Prepr. 2017, 5, e3190v2. [CrossRef]

43. Sharma, H.; Kumar, S. A survey on decision tree algorithms of classification in data mining. Int. J. Sci. Res. (IJSR) 2016, 5, 2094-2097.

44. Géron, A. Hands-On Machine Learning with Scikit-Learn and TensorFlow; O'Reilly Media, Inc.: Sebastopol, CA, USA, 2017.

45. Perez-Lombard, L.; Ortiz, J.; Pout, C. A review on buildings energy consumption information. Energy Build. 2008, 40, 394-398. [CrossRef]

46. Mirakhorli, A.; Bing, D. Occupancy behavior based model predictive control for building indoor climate-A critical review. Energy Build. 2016, 129, 499-513. [CrossRef]

47. Jung, W.; Jazizadeh, F. Human-in-the-loop HVAC operations: A quantitative review on occupancy, comfort, and energy-efficiency dimensions. Appl. Energy 2019, 239, 1471-1508. [CrossRef] 